\title{
Dynamic Equicorrelation Analysis of Financial Contagion: Evidence from Latin America Markets
}

\author{
Roberto Louis Forestal ${ }^{1}$ and Shih Ming $\mathbf{P i}^{2}$
}

\begin{abstract}
This research employs the multivariate autoregressive moving average-generalized autoregressive conditionally heteroscedastic-dynamic equicorrelation (ARMA-GARCHDECO) model to identify contagion among Latin American financial markets during financial turmoil period. We analyze the dynamic conditional correlations among 18 American Depositary Receipts (ADR), 8 Exchange Traded Funds (ETF) and 6 Foreign Exchange Rates (Forex). Our sample includes daily closing prices from April 1, 2014, to January 29, 2021, for Argentina, Brazil, Chile, Colombia, Mexico, and Peru. Results find long-run properties in the volatility of most instruments including those belonging to defensive super sector implying that defensive super sector and basic materials are the most impacted sectors during the last financial crises. We present evidence that in times of economic disruption like in the midst of the COVID-19 pandemic, those financial assets do not act as safe harbor investments since they are relatively more correlated during period of financial crises than in normal periods. Our findings have policy implications and are of interest to practitioners who look a better understanding of the dynamics of spillovers among the behavior of emerging financial assets.
\end{abstract}

JEL classification numbers: C58, D53, G15.

Keywords: Dynamic equicorrelation model, Latin America, American Depositary Receipts, Exchange Traded Funds, Foreign Exchange Rates, ARMA-GARCH.

\footnotetext{
1 Department of Information Management, Chung Yuan Christian University, Taoyuan, Taiwan.

2 College of Business, Chung Yuan Christian University, Taoyuan, Taiwan.
} 


\section{Introduction}

Understanding the volatility dynamics of Latin America financial instruments has received considerable attention following the dramatic Brazilian economic downturn in 2014. Latin America's main market, Brazil, saw a dramatic change when it faced the worst recessions in its history from mid-2014 onward. The problem gradually got worse as the financial situation carries on with its downward spiral, worsened by commodity price shock and political turmoil. This situation has renewed interest in examining the evolution of connectedness among emerging markets and developed economies. Understanding the time-varying connectedness across these markets has several important implications for asset allocations, risk management, policy recommendations and implementation.

Several studies using the dynamic conditional correlation- generalized autoregressive conditional heteroscedasticity (DCC-GARCH) and provide empirical evidence that US stock volatility and weakening credit market conditions induce financial contagion to the Latin America (Hwang, 2014; Rodriguez-Nieto \& Mollick, 2020). Similar methodologies have been used to detect potential contagion among emerging markets. They show that Latin America markets are mostly net volatility receivers (Gamba-Santamaria, Gomez-Gonzalez, HurtadoGuarin, \& Melo-Velandia, 2017; Marçal, Valls Pereira, Martin, \& Nakamura, 2011). Moreover, studies using different methodologies found the interdependence of Latin American assets and call for international portfolio diversification (Costa Correa, Pimenta Júnior, \& Eduardo Gaio, 2018; Esqueda, Luo, \& Jackson, 2015). As this literature indicates, the dynamics of volatility spillover among Latin America markets is an interesting research topic, therefore, it calls for further studies using different approaches and a broader set of datasets. The DCC framework for correlations is a useful modeling tool, however, when the number of test assets becomes large the estimation can become unreliable and even breakdown completely.

The aim of this study is to provide a more comprehensive analysis of static and temporal volatility spillover among Latin America financial instruments, including American Depositary Receipts (ADR), Exchange Traded Funds (ETF) and Foreign Exchange Rates (Forex). We contribute to the existing literature by integrating an ARMA-GARCH-DECO specification with Ling and McAleer (2002) and Engle and Kelly (2012) frameworks to examine conditional spillover among the underlying assets. The Dynamic Equicorrelation (DECO) class of correlation models is aimed to overcome some of computational difficulties of DCC. Regarding ARMA-GARCH, we specifically utilize an $\operatorname{ARMA}(1,1)-\mathrm{GARCH}(1,1)$ specification on returns series to extract the conditional volatility, fat tail, serial correlation, leverage effects, and heteroscedasticity issues. As Danielsson (2011) pointed it out, financial series may be propelled by the presence of volatility clusters and fat tails. Volatility clustering is the observation that "large changes tend to be followed by large changes, of either sign, and small changes tend to be followed by small changes." tendency of large changes in prices of financial assets to cluster together (Mandelbrot, 1997; Tseng \& Li, 2011) while "fat tail" refers to probability distributions with a relatively high probability of extreme outcomes. Those tendencies can be captured using a GARCH framework. To account for structural variation, we divide our sample into three subsamples, i.e. the Brazilian economic crisis period (pre-2017 subsample), the calm period (between 2017 to 2019 subsample) and covi-19 recession period (post-2019 subsample). Consistent with previous research, our results support low level of correlation among the assets under study indicating relatively no interconnectedness among the assets. During the financial turmoil periods, the equicorrelation coefficient among variables is relatively higher compared to calm period. In addition, we found long-run volatility properties among the markets.

The remainder of this article is structured as follows. In section 2 we present previous literature relevant for volatility spillover in Latin America markets. The empirical methodology and the dataset are presented in sections 3 and 4, respectively. The obtained empirical results are discussed in section 5. The final Section 5 concludes the analysis. 


\section{Literature review}

Since the 2008 global financial crisis, an emerging strand of literature employing different datasets and various econometric frameworks focuses on the connectedness dynamics among emerging markets assets such as American depository receipt (ADR), Exchange traded fund (ETF) and Foreign exchange rate (Forex). Hwang (2014) employs DCC-GARCH model to analyze the transmission of the 2008 US financial crisis to four Latin American stock markets. The sample covers daily stock returns from 2006 to 2010 related to specific markets, namely, Merval (Argentina), Bovespa (Brazil), Bolsa de Santiago (Chile) and Bolsa Mexicana de Valores (Mexico). He found evidence of financial contagion by showing that pair-wise conditional correlations are relatively higher and more volatile during the period of crisis. In other words, empirical findings show that stock markets in Argentina, Brazil, and Mexico are heavily affected by the 2008 US financial. Gamba-Santamaria et al. (2017) construct volatility spillover indexes using a DCC-GARCH framework to model the multivariate relationships between US stock markets and four Latin American financial assets. Their results show that Brazil is a net volatility transmitter for most of the sample period, while Chile, Colombia and Mexico are net receivers. The total spillover index is substantially higher between the third quarter of 2008 and the second quarter of 2012, and shock transmission from the US to Latin America substantially increased around the bankruptcy of Lehman Brothers.

Multivariate DCC-GARCH model has also been employed by Rodriguez-Nieto and Mollick (2020) to identify contagion from the USA to the largest developed and emerging markets in the Americas (Argentina, Brazil, Canada, Chile, Colombia, Mexico, and Peru) during the US financial crisis. Their sample considers daily closing prices from January 1, 2002 to December 31,2015 , and includes changes in the general economy's credit risk represented by the TED spread and changes in the US market volatility represented by the CBOE Volatility Index (VIX). Results suggest that increases in VIX have a negative intertemporal and contemporaneous relationship with most of the stock returns, and these relationships increase significantly during the US financial crisis. Moreover, they also find evidence of significant increases in contemporaneous conditional correlations between changes in the TED spread and stock returns. Increases in conditional correlations during the financial crisis are associated with financial contagion from the USA to the Americas. Those findings illustrate that during periods of financial distress, US stock volatility and weakening credit market conditions could promote financial contagion to the Americas. In their article, Marçal et al. (2011) used DCCGARCH model to investigate the existence of contagion among countries on the basis of an analysis of returns for stock indices over the period 1994 to 2003. Results show that contagion spread from the Asian crisis to Latin America, but not in the opposite direction. A possible explanation for Latin America's vulnerability to financial crises lies in the weakness of its economic fundamentals during the period.

Esqueda et al. (2015) employ GARCH-M model to examine the effects of the U.S. investor sentiment on American depository receipts (ADR) premiums by using daily prices from 1995 to 2009. The volatility index (VIX) is used as a proxy for investor expectations about the stock market while liquidity, transaction costs, and domestic and U.S. stock exchange returns are controlled. They find that deviations from the law of one price in ADRs can be partially explained by the lag of the smoothed volatility index. Those findings have important implications for portfolio diversification on emerging markets as investment managers can improve hedging strategies by incorporating known values of the volatility index. In the other hand, Costa Correa et al. (2018) use VAR-MGARCH multivaried skewness models, with diagonal VECH representation to detect and measure the phenomenon of interdependence of ADR indices on the main Latin American capital markets (Brazil, Argentina, Chile and Mexico) and developed (United States, Japan, United Kingdom and France) given the 2008 financial crisis scope. They found that the ADR indices presented greater interdependence with the 
developed countries, compared to the analyzed Latin American equity markets.

In his research paper, Diamandis (2009) uses weekly observations for the period January 1988July 2006 and examines long-run relationships between four Latin America stock markets and a mature stock market that of the US via the autoregressive and moving average representations of a VAR model. The main finding of the analysis suggests that there are significant common permanent components driving the examined stock markets in the long run. Moreover, results also indicate that those five equity markets are partially integrated, implying small long-run benefits from international portfolio diversification since the stock prices adjust very slowly to these common trends. Extending this framework, Esqueda and Jackson (2012) analyze the behavior of 74 American depository receipts (ADR) and exchange rate returns from Argentina, Brazil, Chile, and Mexico by employing seemingly unrelated regressions (SUR) and multivariate regression models (MVRM) during the period May 1994 to May 2009. Results show that ADR prices are determined primarily by the underlying stock, exchange rates, host country index as well as U.S. stock market. Moreover, monitoring the underlying stock and local and host country stock indexes, they find that ADRs generate significant negative abnormal returns during currency crises, due to conversion exposure. Those findings confirm the predominance of the American stock exchanges in terms of ADR price discovery and market integration.

The advantage of our research in comparison with the above studies is the use of ARMAGARCH-DECO specification to test spillover effect among Latin American financial instruments, while prior studies mostly have recourse to multivariate GARCH models to discover US markets contagion to emerging markets.

\section{Methodology}

Fama (1965) suggested that the empirical distribution of stock returns characteristically exhibits a more peaked central part and fatter tail parts compared to the normal distribution assumed by financial theories. Besides those two properties, nonlinear dependence can explain the relationship between multivariate financial data. For example, a non-linear dependence among different assets can be discerned during a financial crisis, where many assets are likely to move together in the same direction depending on certain market conditions (Choudhry, Hassan, \& Shabi, 2015; Escanciano \& Hualde, 2019; Yin \& Wang, 2021). To study those tendencies, let's consider rt returns series for the $\mathrm{t}=1, \ldots, \mathrm{T}$ assets.

\subsection{Conditional Variance}

We define the conditional covariance matrix of all return series as Et-1 [rt rt'] $=\mathrm{Ht}$. We can further decompose $\mathrm{Ht}$ into the following:

$$
H_{t}=D_{t} R_{t} D_{t}
$$

where $D_{t}=\operatorname{diag}\left(\sigma_{i, t}\right)$. Here, $\sigma_{\mathrm{i}, \mathrm{t}}$ is the conditional volatility of return series $i$ and is the $i$ th diagonal entry of $\mathrm{H}_{\mathrm{t}}$. Finally, $\mathrm{R}_{\mathrm{t}}$ is the conditional correlation matrix for the return series. The Autoregressive Moving Average Model-Generalized Autoregressive Conditional Heteroscedasticity-Dynamic Equicorrelation (ARMA-GARCH-DECO) model a la Engle and Kelly (2012) puts specific parametric assumptions on the evolution of $D_{t}$ and $R_{t}$ separately. Each individual return series' conditional variance is displayed as a standard GARCH process. The main advantage of ARCH models is that they can generate accurate models to predict the volatility of financial time series. Conditional variance individual return series can be written as:

$$
\mathrm{E}_{t-1}\left[r_{i, t}^{2}\right]=\sigma_{i, t}^{2}
$$


Following Engle (1982), we use ARMA model to fit the mean and GARCH model to fit the variance. In other words, we utilized the ARMA $(1,1)-\mathrm{GARCH}(1,1)$ models because of their simplicity and reliability. They are given by:

$$
\begin{gathered}
Y_{t}=\mu_{i}+\varphi_{i} Y_{i, t-1}+\varepsilon_{i, t}+\theta_{i} \varepsilon_{i, t-1} \\
\sigma_{i, t}^{2}=\omega_{i}+\alpha_{i} \varepsilon_{i, t-1}^{2}+\beta_{i} \sigma_{i, t-1}^{2}
\end{gathered}
$$

where a given individual return series $\mathrm{Y}_{\mathrm{t}}$ is decomposed into a conditional mean $\left(\mu_{\mathrm{i}}\right)$ containing one lag in both AR and MA terms and a conditional variance $\left(\varepsilon_{t}\right) ; \varphi$ (phi) and $\theta$ (theta) are coefficients to estimate. $\alpha$ (alpha) and $\beta$ (beta) are also model coefficients and are all positive; the constant $\sigma^{2}$ is the unconditional variance of $\varepsilon_{\mathrm{t}}$. We also assume that $\alpha_{\mathrm{i}}+\beta \mathrm{i}<1$ and $\sigma_{\mathrm{it}}{ }^{2}=\omega_{\mathrm{i}} /$ $\left(1-\alpha_{i}-\beta_{i}\right)$. Those are positivity constraints and conditions for existence of the fourth moment of the GARCH (Ling \& McAleer, 2002).

Cai, Tian and Hamori (2016) and Bollerslev (1987) suggest replacing the conditional normal distribution with the conditional Student's t-distribution in order to capture leptokurtosis form of the returns. It takes the following form:

$$
f\left(\varepsilon_{t}\right)=\frac{\Gamma\left[\frac{1+v}{2}\right]}{\sqrt{v \pi} \Gamma\left(\frac{v}{2}\right)}\left[1+\frac{\varepsilon_{t}^{2}}{v}\right]^{-\frac{1+v}{2}}
$$

where $\mathrm{v}$ is the degree of freedom of the $\mathrm{t}$-distribution.

Importantly, the volatility residual vector $\varepsilon_{t}=\left[\varepsilon_{1}, \ldots, \varepsilon_{N, t}\right]$ of our ARMA-GARCH-DECO model will have the same correlation structure as the original return series. We now turn to modeling this correlation structure.

\subsection{Conditional Correlation}

The ARMA-GARCH-DECO model assume a specific parametric form for conditional correlation matrix $\mathrm{R}_{\mathrm{t}}$. More specifically, on a given day the model assumes that all pairwise correlations are identical. Kang, McIver and Yoon (2017) suggest that the correlation matrix $\mathrm{R}_{\mathrm{t}}$ is an equicorrelation matrix and evolves as:

$$
\begin{gathered}
R_{t}=\left(1-\rho_{t}\right) I_{N}+\rho_{t} J_{N} \\
\rho_{t}=\frac{2}{n(n-1)} \sum_{i=1}^{n-1} \sum_{j=i+1}^{n} \frac{q_{i j, t}}{\sqrt{q_{i i, t} q_{j j, t}}} \\
q_{i j, t}=\bar{\rho}_{i, j}+\alpha_{D E C O}\left(\varepsilon_{i, t-1} \varepsilon_{j, t-1}-\bar{\rho}_{i, j}\right)+\beta_{D E C O}\left(q_{i, j, t-1}-\bar{\rho}_{i, j}\right)
\end{gathered}
$$

where $\bar{\rho}_{i, j}$ is the unconditional correlation between $\varepsilon_{\mathrm{i}, \mathrm{t}}$ and $\varepsilon_{\mathrm{j}, \mathrm{t}-1} ; \mathrm{J}_{\mathrm{N}}$ is the $\mathrm{n} \times \mathrm{n}$ matrix of ones, and $\mathrm{I}_{\mathrm{N}} \mathrm{s}$ the $\mathrm{n}$-dimensional identity matrix. This process allows us to represent the degree of co-movement of a group of financial instruments with a single time-varying correlation coefficient. By modeling the univariate return series as individual ARMA-GARCH processes, and their standardized residual series as a DECO process, we form the complete ARMAGARCH-DECO specification. 


\subsection{Estimation}

We estimate the parameters of our ARMA-GARCH-DECO model system using sequential quadratic programming technique in OxMetrics 6.20 software. In order to implement this method, we assume the stacked return series $r_{t}=\left[\begin{array}{ll}r_{1}, t, \ldots, & r_{N}, t\end{array}\right]$ and conditional covariance $\mathrm{H}_{\mathrm{t}}$ followed a Student t-distribution density as explained above. It can be shown that this function can be decomposed into a volatility component and a correlation component, which naturally leads to a two-step estimation procedure \{Doornik, 2002 \#12\}. First, we estimate univariate ARMA-GARCH models to each return series. Next, we use the stacked residuals $\varepsilon_{t}=\left[\varepsilon_{1, t} \ldots, \varepsilon_{N, t}\right]^{\prime}=D_{t}^{-1} r_{t}$, to estimate the correlation parameters $\alpha_{\mathrm{DECO}}$ and $\beta_{\mathrm{DECO}}$ by maximizing the following function:

$$
L_{c}\left(\alpha_{D E C O}, \beta_{D E C O}\right)=\frac{1}{T} \sum_{t}\left(\log \left|R_{t}\right|+\varepsilon_{t}^{\prime} R_{t}^{-1} \varepsilon_{t}-\varepsilon_{t}^{\prime} \varepsilon_{t}^{\prime}\right)
$$

As is similar with the univariate ARMA-GARCH process, the single correlation $\rho_{\mathrm{t}}$ will be stable and mean-reverting so long as $\alpha_{\mathrm{DECO}}>0, \beta_{\mathrm{DECO}}>0, \alpha_{\mathrm{DECO}}+\beta_{\mathrm{DECO}}<1$. The standard restrictions and properties of the univariate ARMA-GARCH models that are used to model each individual return series' volatilities also naturally still hold. Moreover, those estimated parameters may reveal to be consistent estimates in the event that the true correlations evolve as a DCC system, but with much less computational overhead (Cai et al., 2016).

\section{Data}

The sample used in this paper is composed of 3 financial instruments including American Depositary Receipt (ADR), Exchange Traded Fund (ETF) and Foreign Exchange Rate (Forex). Data related to ADR are retrieved from J.P. Morgan's ADR website using filtering criteria such as NYSE as EXCHANGE; Latin America as REGION; and Argentina, Brazil, Chile, Colombia, Mexico and Peru as COUNTRY. 71 relevant ADRs were identified. Out of which 18 are selected based on trading history and market cap. ETF and Forex samples relating to the abovementioned countries have been selected from Yahoo finance based on data availability. All ETFs except ECH are either listed on the NYSE Arca or the NASDAQ Global Market Composite (NASDAQGM). Table 1 details the financial instruments, as well as industry and related country considered in this paper. With a market capitalization of more than US \$43 billion, the Brazilian corporation Ambev is the largest company considered in this study. FMX from Mexico has the second largest market capitalization, of US $\$ 14.9$ billion, making the Beverages/Brewers the most prominent industry under study. Argentina's TGS having a market cap of US $\$ 658$ million is the smallest company while Consumer Defensive remains the most representative sector in this paper. 
Table 1: Categories of financial instruments and related countries

\begin{tabular}{|c|c|c|c|c|c|c|}
\hline Related-country & Type & Symbol & Name & Sector & Industry & $\begin{array}{l}\text { Market cap } \\
\text { (million USD) }\end{array}$ \\
\hline \multirow[t]{5}{*}{ Argentina } & \multirow[t]{3}{*}{ ADR } & TEO & Telecom Argentina & Communication Services & Telecom Services & 1428 \\
\hline & & TGS & Transportadora de Gas del Sur S.A. & Energy & Oil \& Gas Midstream & 658 \\
\hline & & YPF & YPF Sociedad Anónima & Energy & Oil \& Gas Integrated & 2659 \\
\hline & $E T F$ & $A R G T^{l}$ & Global X MSCI Argentina ETF & & & \\
\hline & Forex & $A R S / U S D$ & Argentine Peso to US Dollar Exchange Rate & & & \\
\hline \multirow[t]{9}{*}{ Brazil } & \multirow[t]{6}{*}{ ADR } & ABEV & Ambev S.A. & Consumer Defensive & Beverages-Brewers & 43690 \\
\hline & & $\mathrm{CBD}$ & Companhia Brasileira de Distribuição & Consumer Cyclical & Department Stores & 3723 \\
\hline & & $\mathrm{CIG}$ & Companhia Energética de Minas Gerais & Utilities & Utilities-Diversified & 2544 \\
\hline & & ELP & Companhia Paranaense de Energia - COPEL & Utilities & Utilities-Diversified & 1539 \\
\hline & & SID & Companhia Siderúrgica Nacional & Basic Materials & Steel & 7711 \\
\hline & & TIMB & TIM S.A. & Communication Services & Telecom Services & 5917 \\
\hline & \multirow[t]{2}{*}{ ETF } & $E W Z^{l}$ & iShares MSCI Brazil ETF & & & \\
\hline & & $F B Z^{2}$ & First Trust Brazil AlphaDEX Fund & & & \\
\hline & Forex & $B R L / U S D$ & Brazilian Real to US Dollar Exchange Rate & & & \\
\hline \multirow[t]{5}{*}{ Chile } & \multirow[t]{3}{*}{ ADR } & BSAC & Banco Santander-Chile & Financials Services & Banks-Regional & 9534 \\
\hline & & $\mathrm{CCU}$ & Compañía Cervecerías Unidas S.A. & Consumer Defensive & Beverages-Brewers & 3025 \\
\hline & & ENIA & Enel Américas S.A. & Utilities & Utilities-Regulated Electric & 11435 \\
\hline & $E T F$ & $E C H^{3}$ & iShares MSCI Chile & & & \\
\hline & Forex & $C L P / U S D$ & Chilean Peso to US Dollar Exchange Rate & & & \\
\hline \multirow[t]{4}{*}{ Colombia } & $\mathrm{ADR}$ & CIB & BanColombia S.A. & Financial Services & Banks-Regional & 4367 \\
\hline & \multirow[t]{2}{*}{ ETF } & $C X G^{l}$ & Global X MSCI Colombia ETF & & & \\
\hline & & $I C O L^{I}$ & iShares MSCI Colombia ETF & & & \\
\hline & Forex & $C O P / U S D$ & Colombian Peso to US Dollar Exchange Rate & & & \\
\hline \multirow[t]{6}{*}{ Mexico } & \multirow[t]{4}{*}{ ADR } & FMX & Fomento Económico Mexicano & Consumer Defensive & Beverages-Brewers & 14898 \\
\hline & & IBA & Industrias Bachoco & Consumer Defensive & Farm Products & 2058 \\
\hline & & KOF & Coca-Cola FEMSA & Consumer Defensive & Beverages-Non-Alcoholic & 2322 \\
\hline & & TV & Grupo Televisa & Communication Services & Broadcasting & 4341 \\
\hline & ETF & $E W W^{l}$ & iShares MSCI Mexico ETF & & & \\
\hline & Forex & $M X N / U S D$ & Mexican Peso to US Dollar Exchange Rate & & & \\
\hline \multirow[t]{3}{*}{ Peru } & $\mathrm{ADR}$ & BVN & Compañía de Minas Buenaventura S.A.A. & Basic Materials & Other Precious Metals \& Mining & 2550 \\
\hline & ETF & $E P U^{l}$ & iShares MSCI Peru ETF & & & \\
\hline & Forex & $P E N / U S D$ & Peruvian Nuevo Sol to US Dollar Exchange Rate & & & \\
\hline
\end{tabular}

Note: American depositary receipts (ADR) are all listed on the New York Stock exchange (NYSE). ETF means Exchange-traded fund.

1: Listed on the NYSE Arca; ${ }^{2}$ : Listed on the NASDAQ Global Market Composite (NASDAQGM); ${ }^{3}$ : Listed on the Better Alternative Trading System (BATS)

Source: Yahoo finance (https://finance.yahoo.com/) and J.P Morgan (https://adr.com/dr/drdirectory/drUniverse) 
In addition, our study considers daily data from Yahoo finance for the period starting from April 1, 2014, through January 29, 2021. The choice of this period is based on the devastating impact on the economy of two major crises which are the Brazilian crisis and coronavirus pandemic. This period was further divided into three sub-periods: Brazilian economic crisis (April 1, 2014 - December 30, 2016), calm period (January 3, 2017 - December 31, 2019) and COVID-19 recession (January 2, 2020 - January 29, 2021). From mid-2014 onward, Brazil which is largely dependent on the export of commodities, experienced one of the most intense and prolonged recessions in its economic history charectized by a dramatic drop of GDP score and high unemployment rate (Nassif, 2017; Vartanian \& Garbe, 2019). COVID-19 recession, also known as the Great Lockdown (Ferraresi, Kotsogiannis, Rizzo, \& Secomandi, 2020) is the worst global economic crisis since the Great Depression of the 1930s which has triggered a recession in many economies and regions (Ferraresi et al., 2020; McKibbin \& Fernando, 2020). The situation is taking a heavy toll on emerging stock markets and commodities markets to the point that even safer instruments, such as gold returns, turn negative (Ali, Alam, \& Rizvi, 2020; Topcu \& Gulal, 2020). The sub-sample period named "calm period" considered data between the two above-mentioned crises. Daily returns are computed as 100 times the first difference in the $\log$ of the price level $\mathrm{P}$ of the financial instrument at time $\mathrm{t}$ and time $\mathrm{t}-1$. That is, $\mathrm{rt}=(\log$ $\mathrm{Pt}-\log \mathrm{Pt}-1) \times 100$. Figure 1 plots the daily returns for each financial instrument over the full sample period. These plots reveal that all markets except CLP/USD and COP/USD fell substantially around the global stock market crash beginning on 20 February 2020.
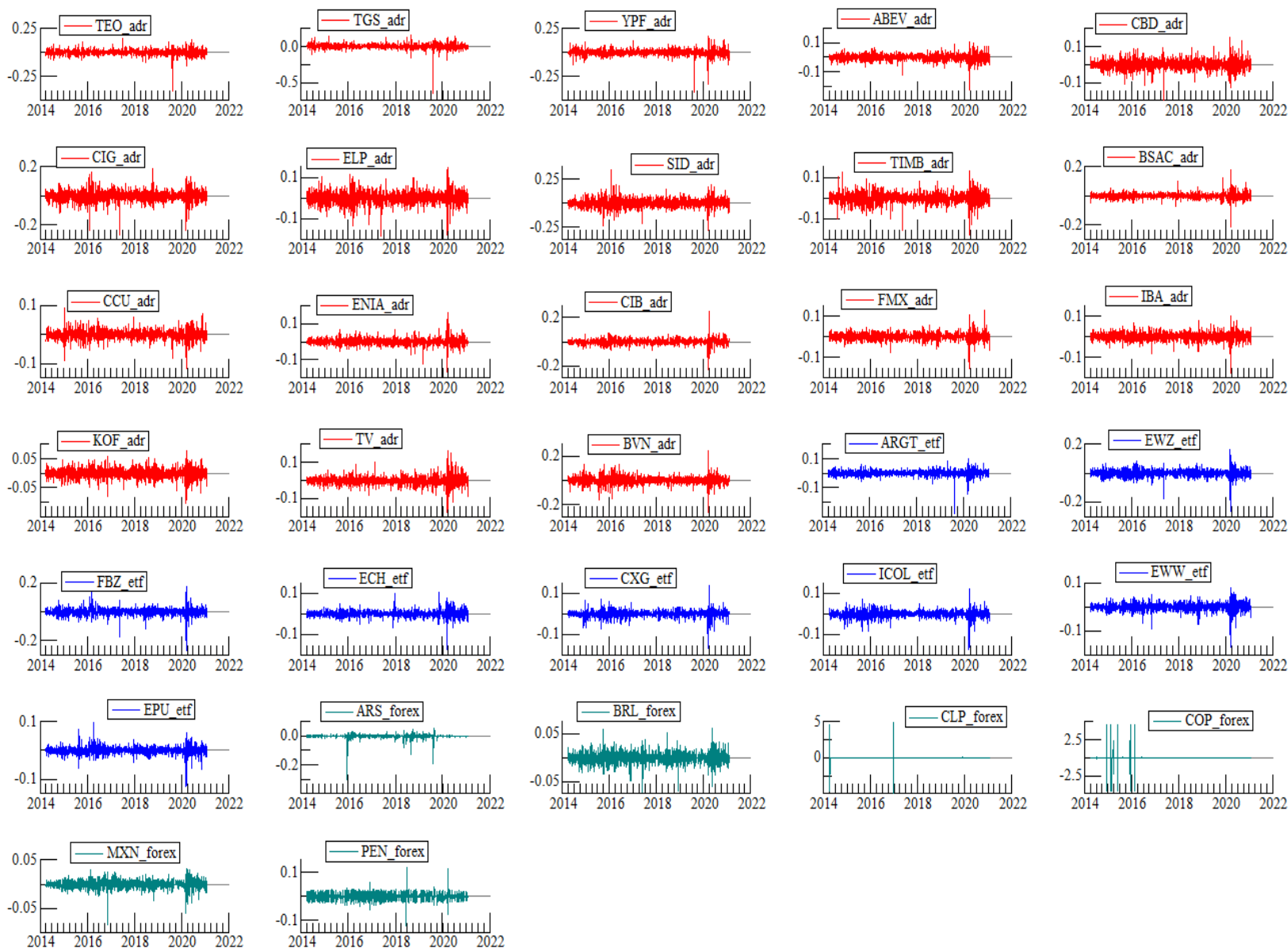

Figure 1: Daily returns of ADR, ETF and Forex (April 1, 2014-January 29, 2021). All returns are the first differences of natural logarithms of the instrument closing price.

Source: Authors' own computations 
Table 2: Sample Size and summary statistics of ADR, ETF and Forex

\begin{tabular}{|c|c|c|c|c|c|c|c|c|c|c|c|c|c|c|}
\hline & \multicolumn{5}{|c|}{ Argentina } & \multicolumn{9}{|c|}{ Brazil } \\
\hline & \multicolumn{3}{|c|}{ ADR } & \multirow{2}{*}{$\begin{array}{c}\text { ETF } \\
\text { ARGT }\end{array}$} & \multirow{2}{*}{$\begin{array}{r}\text { Forex } \\
\text { ARS/USD } \\
\end{array}$} & \multicolumn{6}{|c|}{ ADR } & \multicolumn{2}{|c|}{ ETF } & \multirow{2}{*}{$\begin{array}{r}\text { Forex } \\
\text { BRL/USD }\end{array}$} \\
\hline & TEO & TGS & YPF & & & ABEV & CBD & CIG & ELP & SID & TIMB & EWZ & FBZ & \\
\hline \multicolumn{15}{|c|}{ Panel A. Full sample period - April 1, 2014 through January 29, 2021} \\
\hline Mean & -0.0006 & 0.0004 & -0.0012 & 0.0002 & -0.0014 & -0.0006 & -0.0007 & -0.0006 & -0.0001 & 0.0001 & -0.0004 & -0.0002 & -0.0003 & -0.0005 \\
\hline Std. Dev & 0.0274 & 0.0343 & 0.0332 & 0.0188 & 0.0125 & 0.0226 & 0.0268 & 0.0363 & 0.0303 & 0.0449 & 0.0256 & 0.0250 & 0.0246 & 0.0114 \\
\hline Skewness & -1.7138 & -3.9116 & -1.9456 & -2.4482 & -11.3777 & -0.5819 & -0.2398 & -0.5498 & -0.5154 & 0.1720 & -0.4391 & -1.1867 & -1.3397 & -0.1604 \\
\hline Kurtosis & 32.9269 & 81.5757 & 28.4679 & 36.6783 & 241.6670 & 10.8147 & 6.9621 & 8.5060 & 7.3039 & 8.2190 & 7.6202 & 16.6543 & 21.0915 & 6.8109 \\
\hline Jarque-Bera & 65066.0 & 447125.7 & 47596.9 & 83052.6 & 4121778.0 & 4476.3 & 1142.2 & 2260.6 & 1404.5 & 1961.7 & 1586.0 & 13773.2 & 23985.0 & 1048.8 \\
\hline Probability & .000 & .000 & .000 & .000 & .000 & .000 & .000 & .000 & .000 & .000 & .000 & .000 & .000 & .000 \\
\hline Observations & 1721 & 1721 & 1721 & 1721 & 1721 & 1721 & 1721 & 1721 & 1721 & 1721 & 1721 & 1721 & 1721 & 1721 \\
\hline \multicolumn{15}{|c|}{ Panel B. Brazilian economic crisis period - April 1, 2014 through December 30, 2016} \\
\hline Mean & -0.0001 & 0.0019 & -0.0009 & 0.0002 & -0.0010 & -0.0006 & -0.0014 & -0.0016 & -0.0006 & -0.0004 & -0.0011 & -0.0004 & -0.0005 & -0.0005 \\
\hline Std. Dev & 0.0214 & 0.0265 & 0.0267 & 0.0148 & 0.0132 & 0.0188 & 0.0263 & 0.0379 & 0.0315 & 0.0499 & 0.0263 & 0.0230 & 0.0212 & 0.0116 \\
\hline Skewness & 0.4188 & 0.3982 & 0.0776 & 0.0683 & -17.7293 & -0.0149 & -0.2168 & -0.2924 & -0.1565 & 0.6698 & 0.0102 & 0.0291 & 0.4584 & -0.0744 \\
\hline Kurtosis & 5.9770 & 5.5801 & 4.9344 & 4.2889 & 408.3457 & 3.4884 & 4.3648 & 6.3481 & 4.5229 & 7.9099 & 4.6803 & 3.5627 & 7.4937 & 4.6543 \\
\hline Jarque-Bera & 276.9 & 211.1 & 109.1 & 48.7 & 4794413.0 & 6.9 & 59.4 & 334.5 & 70.0 & 750.1 & 81.8 & 9.3 & 609.1 & 79.9 \\
\hline Probability & .000 & .000 & .000 & .000 & .000 & .031 & .000 & .000 & .000 & .000 & .000 & .01 & .000 & .000 \\
\hline Observations & 695 & 695 & 695 & 695 & 695 & 695 & 695 & 695 & 695 & 695 & 695 & 695 & 695 & 695 \\
\hline \multicolumn{15}{|c|}{ Panel C. Calm period - January 3, 2017 through December 31, 2019} \\
\hline Mean & -0.0006 & -0.0003 & -0.0005 & 0.0002 & -0.0017 & -0.0001 & 0.0004 & 0.0005 & 0.0009 & 0.0001 & 0.0006 & 0.0005 & 0.0003 & -0.0003 \\
\hline Std. Dev & 0.0286 & 0.0384 & 0.0283 & 0.0185 & 0.0140 & 0.0182 & 0.0234 & 0.0304 & 0.0244 & 0.0350 & 0.0199 & 0.0191 & 0.0193 & 0.0100 \\
\hline Skewness & -3.6131 & -6.4493 & -4.0571 & -4.6145 & -4.9306 & -0.5439 & -0.5893 & -0.5620 & -0.4988 & -0.1196 & -0.6394 & -1.1773 & -1.4754 & -0.6461 \\
\hline Kurtosis & 57.1972 & 114.9421 & 64.4213 & 71.8779 & 58.2121 & 7.2623 & 10.7914 & 12.5405 & 8.0263 & 4.7556 & 8.9959 & 13.1289 & 14.1092 & 11.3468 \\
\hline Jarque-Bera & 93921.8 & 398909.9 & 120590.1 & 151721.7 & 98825.0 & 607.9 & 1950.8 & 2899.3 & 825.0 & 98.6 & 1180.8 & 3397.3 & 4150.8 & 2241.2 \\
\hline Probability & .000 & .000 & .000 & .000 & .000 & .000 & .000 & .000 & .000 & .000 & .000 & .000 & .000 & .000 \\
\hline Observations & 754 & 754 & 754 & 754 & 754 & 754 & 754 & 754 & 754 & 754 & 754 & 754 & 754 & 754 \\
\hline \multicolumn{15}{|c|}{ Panel D. COVID-19 recession period - January 2, 2020 through January 29, 2021} \\
\hline Mean & -0.0021 & -0.0013 & -0.0042 & 0.0003 & -0.0013 & -0.0019 & -0.0017 & -0.0011 & -0.0013 & 0.0017 & -0.0017 & -0.0012 & -0.0013 & -0.0011 \\
\hline Std. Dev & 0.0362 & 0.0396 & 0.0543 & 0.0270 & 0.0020 & 0.0375 & 0.0356 & 0.0460 & 0.0401 & 0.0547 & 0.0360 & 0.0399 & 0.0407 & 0.0141 \\
\hline Skewness & 0.0970 & -0.0028 & -1.1395 & -1.1408 & -0.6089 & -0.5485 & 0.1068 & -0.7884 & -0.8143 & -0.6311 & -0.6137 & -1.4397 & -1.5528 & 0.2702 \\
\hline Kurtosis & 4.6813 & 6.1627 & 11.2783 & 9.5265 & 8.6933 & 7.2704 & 5.3860 & 6.8100 & 7.4809 & 7.5047 & 6.6637 & 13.2140 & 14.4269 & 5.0031 \\
\hline Jarque-Bera & 32.5 & 113.4 & 835.5 & 541.7 & 384.2 & 220.3 & 65.0 & 192.7 & 257.6 & 248.0 & 169.2 & 1276.3 & 1589.1 & 48.8 \\
\hline Probability & .000 & .000 & .000 & .000 & .000 & .000 & .000 & .000 & .000 & .000 & .000 & .000 & .000 & .000 \\
\hline Observations & 272 & 272 & 272 & 272 & 272 & 272 & 272 & 272 & 272 & 272 & 272 & 272 & 272 & 272 \\
\hline
\end{tabular}


Table 2 (cont.): Sample Size and summary statistics of ADR, ETF and Forex

\begin{tabular}{|c|c|c|c|c|c|c|c|c|c|c|c|c|c|c|c|c|c|c|}
\hline & \multicolumn{5}{|c|}{ Chile } & \multicolumn{4}{|c|}{ Colombia } & \multicolumn{6}{|c|}{ Mexico } & \multicolumn{3}{|c|}{ Peru } \\
\hline & \multicolumn{3}{|c|}{ ADR } & \multirow{2}{*}{$\begin{array}{c}\text { ETF } \\
\text { ECH }\end{array}$} & \multirow{2}{*}{$\begin{array}{c}\text { Forex } \\
\text { CLP/USD }\end{array}$} & \multirow{2}{*}{$\begin{array}{c}\text { ADR } \\
\text { CIB }\end{array}$} & \multicolumn{2}{|c|}{ ETF } & \multirow{2}{*}{$\begin{array}{c}\text { Forex } \\
\text { COP/USD }\end{array}$} & \multicolumn{4}{|c|}{ ADR } & \multirow{2}{*}{$\begin{array}{c}\text { ETF } \\
\text { EWW }\end{array}$} & \multirow{2}{*}{\begin{tabular}{|c|} 
Forex \\
MXN/USD \\
\end{tabular}} & \multirow{2}{*}{$\begin{array}{l}\text { ADR } \\
\text { BVN }\end{array}$} & \multirow{2}{*}{$\begin{array}{l}\text { ETF } \\
\text { EPU }\end{array}$} & \multirow{2}{*}{\begin{tabular}{|c|} 
Forex \\
PEN/USD \\
\end{tabular}} \\
\hline & BSAC & $\mathbf{C C U}$ & ENIA & & & & CXG & ICOL & & FMX & IBA & KOF & TV & & & & & \\
\hline \multicolumn{19}{|c|}{ Panel A. Full sample period - April 1, 2014 through January 29, 2021 } \\
\hline Mean & -0.0001 & -0.0002 & -0.0001 & -0.0002 & -0.0002 & -0.0003 & -0.0005 & -0.0005 & -0.0005 & -0.0002 & 0.0000 & -0.0005 & -0.0009 & -0.0003 & -0.0003 & -0.0001 & 0.0000 & -0.0002 \\
\hline Std. Dev & 0.0186 & 0.0158 & 0.0188 & 0.0154 & 0.2291 & 0.0234 & 0.0176 & 0.0177 & 0.4002 & 0.0172 & 0.0185 & 0.0168 & 0.0242 & 0.0165 & 0.0083 & 0.0323 & 0.0135 & 0.0129 \\
\hline Skewness & $\begin{array}{l}-0.5055 \\
\end{array}$ & $\begin{array}{l}-0.2563 \\
\end{array}$ & -0.3123 & -0.9924 & 0.0105 & $\begin{array}{l}-0.5788 \\
\end{array}$ & $\begin{array}{l}-1.4579 \\
\end{array}$ & -1.4387 & $\begin{array}{l}-0.0161 \\
\end{array}$ & -0.4341 & $\begin{array}{l}-0.4569 \\
\end{array}$ & -0.4722 & $\begin{array}{l}-0.3562 \\
\end{array}$ & -1.2158 & -1.0403 & -0.2863 & -0.9942 & 0.2189 \\
\hline Kurtosis & 25.3772 & 7.5924 & 16.3225 & 20.0256 & 430.4871 & 24.9393 & 22.3150 & 20.6261 & 124.3696 & 12.1556 & 9.5079 & 6.1608 & 11.5820 & 13.7684 & 11.7211 & 10.5301 & 16.6289 & 16.6145 \\
\hline Jarque-Bera & 35980.6 & 1531.2 & 12756 & 21069 & 13104353 & 34611.8 & 27362 & 22872 & 1056305.0 & 6065.1 & 3096.9 & 780.3 & 5317.7 & 8739.1 & 5764.3 & 4089.5 & 13603 & 13305.2 \\
\hline Probability & .000 & .000 & .000 & .000 & .000 & .000 & .000 & .000 & .000 & .000 & .000 & .000 & .000 & .000 & .000 & .000 & .000 & .000 \\
\hline Observations & 1721 & 1721 & 1721 & 1721 & 1721 & 1721 & 1721 & 1721 & 1721 & 1721 & 1721 & 1721 & 1721 & 1721 & 1721 & 1721 & 1721 & 1721 \\
\hline \multicolumn{19}{|c|}{ Panel B. Brazilian economic crisis period - April 1, 2014 through December 30, 2016} \\
\hline Mean & -0.0001 & -0.0001 & 0.0000 & -0.0003 & -0.0002 & -0.0006 & -0.0010 & -0.0010 & -0.0009 & -0.0003 & 0.0002 & -0.0007 & -0.0007 & -0.0005 & -0.0006 & -0.0002 & 0.0000 & -0.0003 \\
\hline Std. Dev & 0.0143 & 0.0157 & 0.0155 & 0.0119 & 0.3606 & 0.0197 & 0.0168 & 0.0177 & 0.6299 & 0.0154 & 0.0168 & 0.0158 & 0.0170 & 0.0137 & 0.0078 & 0.0370 & 0.0132 & 0.0131 \\
\hline Skewness & -0.0339 & 0.0238 & 0.3364 & -0.0257 & 0.0074 & 0.0495 & -0.1282 & -0.2750 & -0.0084 & -0.3428 & 0.0051 & -0.1550 & -0.1189 & -0.7964 & -1.6058 & 0.0702 & 0.7961 & -0.0724 \\
\hline Kurtosis & 3.9977 & 6.9083 & 4.3924 & 4.3860 & 174.1797 & 4.3722 & 5.0157 & 5.4694 & 50.2648 & 4.3305 & 4.1331 & 4.7336 & 5.4726 & 8.5426 & 20.8082 & 4.5088 & 9.2644 & 4.0380 \\
\hline Jarque-Bera & 29.0 & 442.4 & 69.3 & 55.7 & 848551.2 & 54.8 & 119.6 & 185.4 & 64691.8 & 64.9 & 37.2 & 89.8 & 178.7 & 963.1 & 9482.3 & 66.5 & 1209.8 & 31.8 \\
\hline Probability & .000 & .000 & .000 & .000 & .000 & .000 & .000 & .000 & .000 & .000 & .000 & .000 & .000 & .000 & .000 & .000 & .000 & .000 \\
\hline Observations & 695 & 695 & 695 & 695 & 695 & 695 & 695 & 695 & 695 & 695 & 695 & 695 & 695 & 695 & 695 & 695 & 695 & 695 \\
\hline \multicolumn{19}{|c|}{ Panel C. Calm period - January 3, 2017 through December 31, 2019} \\
\hline Mean & 0.0001 & -0.0001 & 0.0004 & -0.0002 & -0.0001 & 0.0005 & 0.0001 & 0.0001 & -0.0002 & 0.0003 & 0.0001 & -0.0001 & -0.0008 & 0.0000 & 0.0001 & 0.0004 & 0.0002 & 0.0001 \\
\hline Std. Dev & 0.0144 & 0.0124 & 0.0159 & 0.0127 & 0.0093 & 0.0160 & 0.0111 & 0.0108 & 0.0100 & 0.0138 & 0.0169 & 0.0144 & 0.0195 & 0.0131 & 0.0070 & 0.0204 & 0.0092 & 0.0129 \\
\hline Skewness & 0.9098 & -0.3496 & -0.5258 & 0.9095 & 0.4146 & 0.0634 & -0.2100 & -0.1619 & -0.6169 & -0.0471 & -0.1289 & -0.0684 & -0.0926 & -0.4623 & -0.2905 & -0.1415 & -0.3010 & -0.0975 \\
\hline Kurtosis & 14.4176 & 6.3477 & 9.7499 & 14.4533 & 7.9581 & 4.1937 & 4.1792 & 4.2420 & 4.7571 & 4.3358 & 4.8018 & 4.3409 & 5.5365 & 5.6703 & 4.2083 & 5.1166 & 4.3990 & 23.5539 \\
\hline Jarque-Bera & 4199.6 & 367.4 & 1466.1 & 4225.1 & 793.9 & 45.3 & 49.2 & 51.8 & 144.8 & 56.3 & 104.1 & 57.1 & 203.2 & 250.9 & 56.5 & 143.3 & 72.9 & 13273.6 \\
\hline Probability & .000 & .000 & .000 & .000 & .000 & .000 & .000 & .000 & .000 & .000 & .000 & .000 & .000 & .000 & .000 & .000 & .000 & .000 \\
\hline Observations & 754 & 754 & 754 & 754 & 754 & 754 & 754 & 754 & 754 & 754 & 754 & 754 & 754 & 754 & 754 & 754 & 754 & 754 \\
\hline \multicolumn{19}{|c|}{ Panel D. COVID-19 recession period - January 2, 2020 through January 29, 2021} \\
\hline Mean & -0.0005 & -0.0005 & -0.0014 & -0.0004 & -0.0001 & -0.0016 & -0.0011 & -0.0011 & -0.0004 & -0.0012 & -0.0009 & -0.0012 & -0.0016 & -0.0004 & -0.0005 & -0.0015 & -0.0003 & -0.0005 \\
\hline Std. Dev & 0.0332 & 0.0231 & 0.0305 & 0.0265 & 0.0090 & 0.0420 & 0.0300 & 0.0292 & 0.0112 & 0.0271 & 0.0255 & 0.0242 & 0.0440 & 0.0275 & 0.0122 & 0.0442 & 0.0217 & 0.0126 \\
\hline Skewness & -0.7367 & -0.3667 & -0.2760 & -1.5026 & -0.3062 & -0.5698 & -1.7273 & -1.7818 & -1.5379 & -0.4351 & -0.9472 & -0.7976 & -0.2950 & -1.2453 & -0.8340 & -0.7036 & -1.8843 & 2.0354 \\
\hline Kurtosis & 14.1660 & 5.4122 & 12.2267 & 12.0369 & 3.7086 & 14.3743 & 15.3398 & 15.4838 & 11.1347 & 10.3594 & 11.9586 & 5.3733 & 5.8753 & 8.9999 & 5.8821 & 12.7030 & 12.0138 & 33.7369 \\
\hline Jarque-Bera & 1437.6 & 72.0 & 968.3 & 1027.9 & 9.9 & 1481.0 & 1861.0 & 1910.2 & 857.2 & 622.4 & 950.2 & 92.7 & 97.6 & 478.3 & 125.7 & 1089.4 & 1081.8 & 10895.0 \\
\hline Probability & .000 & .000 & .000 & .000 & .007 & .000 & .000 & .000 & .000 & .000 & .000 & .000 & .000 & .000 & .000 & .000 & .000 & .000 \\
\hline Observations & 272 & 272 & 272 & 272 & 272 & 272 & 272 & 272 & 272 & 272 & 272 & 272 & 272 & 272 & 272 & 272 & 272 & 272 \\
\hline
\end{tabular}




\section{Empirical Results}

Table 2 described the sample size and summary statistics of our variables. Latin American financial instruments except TGS, ARGT and SID have negative daily mean returns during the full sample period. The average returns range from -0.0014 (ARS/USD) to 0.0004 (TGS). Standard deviations range from 0.0083 (MXN/USD) to 0.4002 (COP/USD). In other words, COP/USD following by CLP/USD, SID and CIG are more significantly volatile than any other instrument. Skewness describing the asymmetry of the normal distribution shows that all instruments except SID and CLP/USD have negative (left skewness) value, indicating a greater probability to generate negative return outcomes. Kurtosis coefficient measuring the peakedness of the distribution imply that all variables have leptokurtic distribution (positive excess kurtosis) and shows evidence of fat tails in all markets. In addition, the Jarque-Bera test strongly rejects the normality of returns series. The means are mostly negative during the Brazilian economic crisis. The mean returns range from -0.0016 (CIG) to 0.0019 (TGS). COP/USD, CLP/USD, SID and CIG having respectively a standard deviation of 0.6299, 0.3606 and 0.0499 are the most volatile instruments while MXN/USD is the safest one. In addition, all variables are positively skewed and have positive excess kurtosis while the Jarque-Bera test strongly rejects the normality confirming that the distribution has fatter tails.

As expected, the average value of financial instruments ranging from -0.0017 (ARS/USD) and 0.0009 (ELP) are more likely positive during the calm period. Higher standard deviation scores 0.0384 (STGS), 0.035 (SID) and 0.0304 (CIG) makes those variable the most volatile instruments while MXN/USD and EPU are the less volatile one during this period. Moreover, variables mostly have a long-left tail and positive excess kurtosis while the test of Jarque-Bera strongly rejects the normality distribution assumption. On the other hand, during the COVID19 lock-down period, the mean return of financial instruments is mostly negative and range from -0.0042 (YPF) and 0.0017 (SID). ARS/USD with a standard deviation of 0.0020 is the safest instrument while SID having a standard deviation of 0.0547 appears to be the most volatile among variables. Additionally, returns are mostly negatively skewed while their kurtosis values are greater than 3 (leptokurtic distributions), which means that the probability of extreme return is very high. The Jarque-Bera statistic for residual normality shows that the returns of those instruments are under a non-normal distribution assumption.

Table 3 illustrates the use of Augmented Dickey-Fuller (ADF) test to evaluate whether our series have unit root or not. This test indicates that the return of the instruments under study all reject the null hypothesis of a unit root at $1 \%$ level of significance, meaning that the return series are stationary according to the ADF. Based on our model specification, we use one lag value of both the AR and MA parameters to select the models. But the Lagrange Multiplier (LM) test returns serial correlation problem in more about one-third of the markets. This study also used the ARCH-LM process to test the ARCH effect and eliminate heteroscedasticity in the volatility of the data; the test illustrates that the GARCH $(1,1)$ models can be applied in the returns. It showed that all instruments except BRL-USD, TV and EWW are now free of heteroscedasticity problems with insignificant values starting from 0.0024 to 2.3555 . In other words, the test results suggest no autoregressive conditional heteroscedasticity for each sample in the GARCH-ARMA models. 
Table 3: Summary Statistics of Unit Root, LM, and ARMA-LM tests for ADR, ETF and Forex

\begin{tabular}{|c|c|c|c|c|c|c|c|c|}
\hline ADR/ETF/Forex & ADF & ARMA & AIC & LM & ARCH-LM & GARCH & AIC & ARCH-LM \\
\hline TEO & \begin{tabular}{l|l|l|}
-22.7009 & $* * *$ \\
\end{tabular} & $(1,1)$ & -4.3565 & 3.6775 & $7.6583^{* *}$ & $(1,1)$ & -4.5632 & 0.5204 \\
\hline TGS & $-17.6838 \mid * * *$ & $(1,1)$ & -3.9071 & 1.6352 & \begin{tabular}{l|l}
0.4679 & \\
\end{tabular} & $(1,1)$ & -4.1385 & 0.7217 \\
\hline YPF & \begin{tabular}{ll|l}
-13.7109 & $* * *$ \\
\end{tabular} & $(1,1)$ & -3.9716 & 3.9602 & 73.4609 *** & $(1,1)$ & -4.3020 & 2.3555 \\
\hline ARGT & $-12.4962 \mid * * *$ & $(1,1)$ & -5.1049 & $4.9841 *$ & $15.9462 * * *$ & $(1,1)$ & -5.3538 & 0.0888 \\
\hline ARS/USD & \begin{tabular}{|l|l|l|}
-10.1020 & $* * * *$ \\
\end{tabular} & $(1,1)$ & -5.9305 & \begin{tabular}{l|l}
1.8942 \\
\end{tabular} & \begin{tabular}{|l|l|}
2.4684 & \\
\end{tabular} & $(1,1)$ & -6.7150 & 0.0341 \\
\hline ABEV & $-17.8151 * * *$ & $(1,1)$ & -4.7477 & 3.0643 & $100.7680^{* * *}$ & $(1,1)$ & -4.9467 & 0.4308 \\
\hline CBD & $-17.6900 * * *$ & $(1,1)$ & -4.3966 & $4.9946 *$ & 80.6383 *** & $(1,1)$ & -4.4895 & 0.9629 \\
\hline CIG & $-14.9660 * * *$ & $(1,1)$ & -3.7955 & 1.0493 & $113.8730^{* * *}$ & $(1,1)$ & -3.9752 & 0.3140 \\
\hline ELP & \begin{tabular}{ll|l}
$-13.0503 * * *$ \\
\end{tabular} & $(1,1)$ & -4.1587 & 0.5219 & $157.54766^{* * *}$ & $(1,1)$ & -4.3359 & 0.7157 \\
\hline SID & $-42.6480 * * *$ & $(1,1)$ & -3.3670 & 2.8888 & 77.7574 *** & $(1,1)$ & -3.5381 & 1.5426 \\
\hline TIMB & $-10.4940 * * *$ & $(1,1)$ & -4.4915 & 1.5255 & $107.2668 * * *$ & $(1,1)$ & -4.6209 & 0.3706 \\
\hline EWZ & $-12.9064 * * *$ & $(1,1)$ & -4.5663 & 7.1450 *** & $453.21877^{* * *}$ & $(1,1)$ & -4.8390 & 0.0280 \\
\hline FBZ & $-11.7363 * * *$ & $(1,1)$ & -4.5868 & 3.0096 & 296.9472 *** & $(1,1)$ & -4.8609 & 0.3253 \\
\hline BRL/USD & $-46.8240 * * *$ & $(1,1)$ & -6.1293 & \begin{tabular}{l|l}
0.8109 \\
\end{tabular} & $66.0424 * * *$ & $(1,1)$ & -6.2426 & $6.3509 * *$ \\
\hline BSAC & \begin{tabular}{l|l|l|l|}
-24.5172 & $* * *$ \\
\end{tabular} & $(1,1)$ & -5.1538 & 6.5253 ** & 284.0638 *** & $(1,1)$ & -5.4820 & $\begin{array}{l}0.4868 \\
\end{array}$ \\
\hline $\mathrm{CCU}$ & $-19.5804 * * *$ & $(1,1)$ & -5.4543 & \begin{tabular}{l|l}
3.9750 \\
\end{tabular} & 60.2379 *** & $(1,1)$ & -5.5985 & 1.2801 \\
\hline ENIA & $-15.0977 * * *$ & $(1,1)$ & -5.1071 & $6.8630 * *$ & $303.7472 * * *$ & $(1,1)$ & -5.3538 & 0.4182 \\
\hline $\mathrm{ECH}$ & \begin{tabular}{l|l|l|}
$-14.8008 * * *$ \\
\end{tabular} & $(1,1)$ & -5.5070 & $25.5288 * * *$ & 392.8134 *** & $(1,1)$ & -5.8371 & 1.4728 \\
\hline CLP-USD & \begin{tabular}{|l|l|l|}
-17.4017 & $* * *$ \\
\end{tabular} & $(1,1)$ & -0.7686 & \begin{tabular}{l|l|}
1.0417 & \\
\end{tabular} & \begin{tabular}{|l|l|}
0.0035 & \\
\end{tabular} & $(1,1)$ & -4.2205 & 0.0024 \\
\hline CIB & $-11.2218 * * *$ & $(1,1)$ & -4.6824 & 2.7944 & $328.5083 * * *$ & $(1,1)$ & -5.0838 & 1.3534 \\
\hline $\mathrm{CXG}$ & $\begin{array}{l}-13.7790 * * * \\
\end{array}$ & $(1,1)$ & -5.2719 & 2.8293 & $371.8041^{* * *}$ & $(1,1)$ & -5.7093 & 0.2858 \\
\hline ICOL & $-13.6408 * * *$ & $(1,1)$ & -5.2569 & $5.6125 *$ & $292.4970^{* * * *}$ & $(1,1)$ & -5.6227 & 0.1640 \\
\hline COP/USD & $-15.0608 * * *$ & $(1,1)$ & 0.3163 & $23.7637 * * *$ & \begin{tabular}{l|l}
0.0090 \\
\end{tabular} & $(1,1)$ & 0.0749 & 0.0107 \\
\hline FMX & \begin{tabular}{l|l|l|}
-11.9802 & $* * *$ \\
\end{tabular} & $(1,1)$ & -5.2901 & $6.6209 * *$ & 156.8076 *** & $(1,1)$ & -5.4836 & 1.6338 \\
\hline IBA & $-44.7490 * * *$ & $(1,1)$ & -5.1454 & \begin{tabular}{l|l}
0.8246 \\
\end{tabular} & 74.0269 *** & $(1,1)$ & -5.2596 & 1.5404 \\
\hline $\mathrm{KOF}$ & $-43.4363 * * *$ & $(1,1)$ & -5.3292 & $\begin{array}{l}0.4167 \\
\end{array}$ & $164.65311^{* * *}$ & $(1,1)$ & -5.4697 & 0.0639 \\
\hline TV & $-14.4197 * * *$ & $(1,1)$ & -4.6033 & 15.9266 *** & 191.4253 *** & $(1,1)$ & -4.9286 & 7.3836 ** \\
\hline EWW & $-9.3558 * * *$ & $(1,1)$ & -5.3721 & \begin{tabular}{l|l}
2.7377 \\
\end{tabular} & $298.69133^{* * *}$ & $(1,1)$ & -5.7241 & $5.2137 *$ \\
\hline MXN/USD & $-40.6380 * * *$ & $(1,1)$ & -6.7360 & \begin{tabular}{l|l}
0.0551 & \\
\end{tabular} & 63.57915 *** & $(1,1)$ & -6.9865 & 1.0562 \\
\hline BVN & $\begin{array}{l}-14.3690 * * * \\
\end{array}$ & $(1,1)$ & -4.0316 & 6.8448 ** & $186.26733^{* * *}$ & $(1,1)$ & -4.2952 & 0.6160 \\
\hline EPU & $\begin{array}{l}-9.9301 * * * \\
\end{array}$ & $(1,1)$ & -5.7813 & 8.3776 ** & 359.2711 *** & $(1,1)$ & -6.1554 & 1.1079 \\
\hline PEN/USD & $-14.5425 * * *$ & $(1,1)$ & -6.1755 & 1.4365 & $\left.10.1496\right|^{* * *}$ & $(1,1)$ & -6.1895 & 0.0662 \\
\hline
\end{tabular}

ADF: t-statistic for the Augmented Dickey-Fuller test with a constant and trend at the level. ARMA: Autoregressive Moving Average model order, AIC: Akaike information criterion; LM:

Breusch-Godfrey serial correlation test.; ARCH-LM: Engle's LM test for ARCH effects; GARCH: Generalized Autoregressive Conditional Heteroscedasticity model order.

Source: Authors' own computations 
Table 4(a): Summary Statistics of multivariate ARMA-GARCH-DECO Model (full sample period)

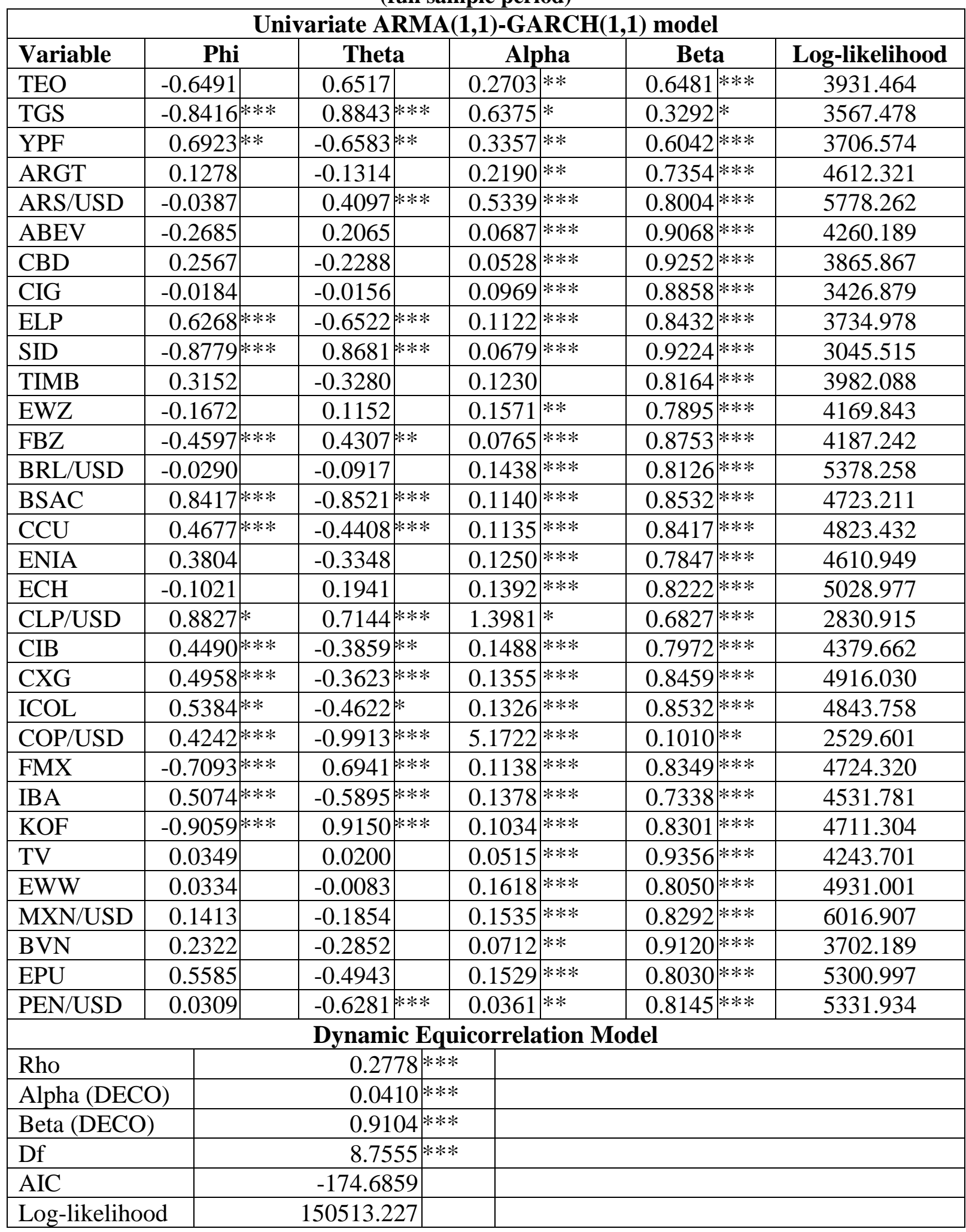

Note: $*, * *$ and $* * *$ are significance at 10,5 and $1 \%$ levels. Df refers to degrees of freedom and AIC means Akaike information criterion.

Source: Authors' own computations 
In Table 4(a), we estimate ARMA-GARCH-DECO models for the overall sample. The sample mean of squared residuals was used to start recursion. The positivity constraint for the GARCH $(1,1)$ and the condition for existence of the fourth moment of the GARCH are both observed in instruments belonging to defensive super sector (ABEV, CBD, CIG, ELP, CCU, ENIA, FMX, IBA, KOF, TV), financial services and basic materials sectors (TEO, SID, TIMB, BVN, BSAC, CIB), ETF (EWZ, FBZ, ECH, CXG, EWW, EPU) and forex (BRL/USD, PEN/USD). Those constraints are given by alpha $>0$, alpha/ $(1-$ beta $) \geq 0$ and alpha + beta $<$ 1 as proposed by Doornik et al. (2002) and Ling and McAleer (2002). The coefficient $\alpha$ (alpha) captures the influence of new shocks on volatility while the parameter $\beta$ (beta), measures persistence of volatility shocks. Results show that the volatility persistence of those assets is not very long in this period, and there is a certain degree of "volatility clustering phenomenon". In the other hand, the constraint of stationarity is observed in assets coming from exchanged traded fund (ARGT, ICOL) and foreign exchange rate (CLP/USD, COP/USD, MXN/USD). However, the condition for existence of the fourth moment of the GARCH is not observed. That constraint is over 1, meaning that there is a long-lasting volatility in those markets. Other results show that the positivity constraint for the GARCH $(1,1)$ and the condition for existence of the fourth moment of the GARCH are not observed in ARS/USD. This constraint equals 2.35067>1. Dynamic Equicorrelation Model coefficients are all statistically significant. Rho having a score of 0.2778 show the level of correlation among the assets under study. Alpha (DECO) measures the short-run volatility impact while Beta (DECO) rate the long-run volatility effect. The results show that the model captures the long-run volatility impact among the markets with a score close to 1 (0.9104).

Table 4(b) presents the estimation of ARMA-GARCH-DECO models for the Brazilian crisis period. Results show that when $\theta$ (theta) coefficient is negative, $\varphi$ (phi) is positive, and in reverse. The stationarity condition is that this factor be less than the unit in absolute value. Furthermore, the positivity constraint for the GARCH $(1,1)$ and the condition for existence of the fourth moment of the GARCH are both observed in assets derived from communication services and energy sectors (TEO, TGS, YPF, TIMB), defensive super sector (ABEV, CBD, CIG, ELP, CCU, ENIA, FMX, IBA, KOF, TV), financial services and basic materials (BSAC, CIB, BVN), Exchange-traded fund (ARGT, EWZ, FBZ, ECH, ICOL) and forex (BRL/USD). This shows that Telecom Services and utilities industries have the most volatile assets during this period. In addition, the coefficient $\alpha$ (alpha) and $\beta$ (beta) are positive and statistically significant for those returns (except for TEO, TGS, CIB and TV) implying that economic shocks especially those of external do have long standing effects on those markets. Similarly, the positivity constraint for the GARCH $(1,1)$ is observed in ETF (CXG, EWW, EPU) and forex (ARS/USD, CLP/USD). However, the condition for existence of the fourth moment of the GARCH is not observed. That constraint is over 1. For returns such as CXG, EWW and EPU, value of $\beta$ is close to 1 , indicating that old shocks tend to persist, instead of dying out quickly. Other results show that the positivity constraint for the GARCH $(1,1)$ is not observed while the condition for existence of the fourth moment of the GARCH is observed in PEN/USD. That constraint equals 0.942848 and should be $<1$. Its negative and statistically significant $\beta$ implies that economic shocks don't have any effects on this exchange rate volatility. Figure 2 plots the conditional variance. The plot seems to indicate that the volatility increases as time passes for most of the assets which is confirmed in Figure 3 drawing the conditional correlation during that crisis period. During the Brazilian crisis period, the equicorrelation coefficient, with a score of 0.3160 , is higher than that of the overall sample, supporting the predictions of Hwang (2014) stating that the total spillover is substantially higher in period of financial turmoil. Beta (DECO) summarizes the long-run volatility impact among the markets with a score close to 1. Alpha (DECO) measuring the short-term volatility effect is low but statistically not significant. 
Table 4(b): Summary Statistics of multivariate ARMA-GARCH-DECO Model (Brazilian crisis period)

\begin{tabular}{|c|c|c|c|c|c|c|}
\hline \multicolumn{7}{|c|}{ Univariate ARMA(1,1)-GARCH $(1,1)$ model } \\
\hline Variable & Phi & \multicolumn{2}{|c|}{ Theta } & Alpha & Beta & Log-likelihood \\
\hline TEO & $-0.7324 \mid * * *$ & \multicolumn{2}{|c|}{0.7093 **** } & 0.0322 & 0.9623 *** & 1703.454 \\
\hline TGS & $0.3993 * *$ & \multicolumn{2}{|c|}{$-0.3440 *$} & 0.0932 & $0.8930^{* * * *}$ & 1576.057 \\
\hline YPF & 0.3079 & \multicolumn{2}{|l|}{-0.2230} & $0.1394 * *$ & $0.7683^{* * * *}$ & 1564.665 \\
\hline ARGT & 0.2132 & \multicolumn{2}{|l|}{-0.1478} & $\begin{array}{l}0.1047 \\
\end{array}$ & $0.7430^{* * * *}$ & 1957.155 \\
\hline ARS/USD & 0.0883 & \multicolumn{2}{|c|}{$0.4653 * * *$} & \begin{tabular}{l|l}
2.0783 \\
\end{tabular} & \begin{tabular}{l|l}
0.4693 & \\
\end{tabular} & 2304.336 \\
\hline $\mathrm{ABEV}$ & 0.8038 *** & \multicolumn{2}{|c|}{$-0.8408 * * *$} & 0.0654 *** & $0.8901^{* * * *}$ & 1794.200 \\
\hline CBD & \begin{tabular}{l|l}
0.2734 \\
\end{tabular} & \multicolumn{2}{|c|}{\begin{tabular}{l|l}
-0.1628 \\
\end{tabular}} & 0.0674 *** & $0.9269 * * *$ & 1583.107 \\
\hline $\mathrm{CIG}$ & $-0.7810 * * *$ & \multicolumn{2}{|c|}{$0.7968 * * *$} & 0.0991 ** & $0.8658 * * *$ & 1336.572 \\
\hline ELP & $-0.8315 * * *$ & \multicolumn{2}{|c|}{0.8093 *** } & \begin{tabular}{l|l|l|}
0.0948 & $* *$ \\
\end{tabular} & $0.8552 * * *$ & 1446.866 \\
\hline SID & \begin{tabular}{l|l}
-0.0252 \\
\end{tabular} & \multicolumn{2}{|c|}{\begin{tabular}{l|l}
0.0833 \\
\end{tabular}} & $0.0709 * * *$ & $0.9299 * * *$ & 1160.283 \\
\hline TIMB & -0.2252 & \multicolumn{2}{|l|}{0.2680} & \begin{tabular}{l|l}
0.1527 & \\
\end{tabular} & $0.6359 * * *$ & 1552.348 \\
\hline EWZ & 0.0575 & -0.0289 & & $0.0764 * * *$ & $0.9024 * * *$ & 1665.106 \\
\hline FBZ & -0.3721 & 0.3849 & & $\begin{array}{l}0.0258 * * \\
\end{array}$ & $0.9581^{* * *}$ & 1702.729 \\
\hline BRL/USD & $0.4808 * *$ & $-0.5500 *$ & & $0.1069 * *$ & $0.8583 * * *$ & 2140.096 \\
\hline BSAC & \begin{tabular}{l|l}
0.2465 \\
\end{tabular} & -0.2270 & & 0.0583 *** & $0.9069 * * *$ & 1985.300 \\
\hline $\mathrm{CCU}$ & 0.3076 & -0.2364 & & $0.1221 * *$ & $0.8134 * * *$ & 1934.591 \\
\hline ENIA & 0.0508 & -0.0089 & & $\begin{array}{l}0.0798 * * * \\
\end{array}$ & $0.8791^{* * * *}$ & 1934.690 \\
\hline $\mathrm{ECH}$ & -0.0179 & 0.1493 & & $0.0969 * *$ & $0.8088^{* * * *}$ & 2119.831 \\
\hline CLP/USD & $1.0822 *$ & $0.7481^{*}$ & $* * *$ & $2.4055 *$ & $0.6620 * * *$ & 929.029 \\
\hline CIB & $0.4629 * * *$ & $-0.3511 *$ & $* * *$ & 0.0864 & $0.8789 * * *$ & 1790.916 \\
\hline $\mathrm{CXG}$ & $0.3051^{* * * *}$ & -0.1184 & & $0.1049 * * *$ & $0.8865^{* * *}$ & 1923.057 \\
\hline ICOL & $\begin{array}{ll}0.1402 \\
\end{array}$ & -0.0320 & & 0.0385 ** & $0.9470 * * *$ & 1841.995 \\
\hline COP-USD & $\mathrm{nc}$ & $\mathrm{nc}$ & & nc & nc & $\mathrm{nc}$ \\
\hline FMX & \begin{tabular}{l|l}
0.3083 \\
\end{tabular} & -0.2684 & & $\begin{array}{l}0.0592 \mid * * \\
\end{array}$ & $0.7930 * * *$ & 1925.826 \\
\hline IBA & $0.6691 * * *$ & -0.7193 * & $* * *$ & 0.1562 *** & $0.6768 * * *$ & 1870.116 \\
\hline KOF & $-0.8965 \mid * * *$ & $0.9235^{*}$ & $* * *$ & $0.1731 * * *$ & $0.3446 * * *$ & 1916.082 \\
\hline TV & \begin{tabular}{l|l}
-0.3800 \\
\end{tabular} & 0.4772 & & \begin{tabular}{l|l}
0.0120 & \\
\end{tabular} & $0.9812^{* * * *}$ & 1855.323 \\
\hline EWW & 0.0957 & 0.0007 & & $0.1430 * * *$ & $0.8367 * * *$ & 2060.331 \\
\hline MXN/USD & nc & $\mathrm{nc}$ & & nc & nc & $\mathrm{nc}$ \\
\hline $\mathrm{BVN}$ & $\begin{array}{l}0.3192 \\
\end{array}$ & $-0.3794 *$ & $* * *$ & $0.0505 * * *$ & $0.9311^{* * *}$ & 1335.331 \\
\hline EPU & $\begin{array}{l}0.0485 \\
\end{array}$ & 0.0837 & & 0.1149 *** & $0.8770^{* * * * *}$ & 2106.255 \\
\hline PEN/USD & 0.0098 & -0.6265 * & $* * *$ & 0.0284 *** & $-0.9985 * * *$ & 2148.409 \\
\hline & & Dynamic $F$ & Equ & rrelation $\mathrm{M}$ & & \\
\hline Rho & $0.3160^{*}$ & & & & & \\
\hline $\begin{array}{l}\text { Alpha } \\
\text { (DECO) }\end{array}$ & 0.0117 & & & & & \\
\hline Beta (DECO) & $0.9868^{*}$ & & & & & \\
\hline Df & $9.4516^{*}$ & & & & & \\
\hline AIC & -172.1865 & & & & & \\
\hline $\begin{array}{l}\text { Log- } \\
\text { likelihood }\end{array}$ & 60030.795 & & & & & \\
\hline
\end{tabular}

Note: *,** and *** are significance at 10,5 and $1 \%$ levels; nc means that the tests are not reported since there is no convergence (no improvement in line search) using numerical derivatives. Df refers to degrees of freedom and AIC means Akaike information criterion.

Source: Authors' own computations 

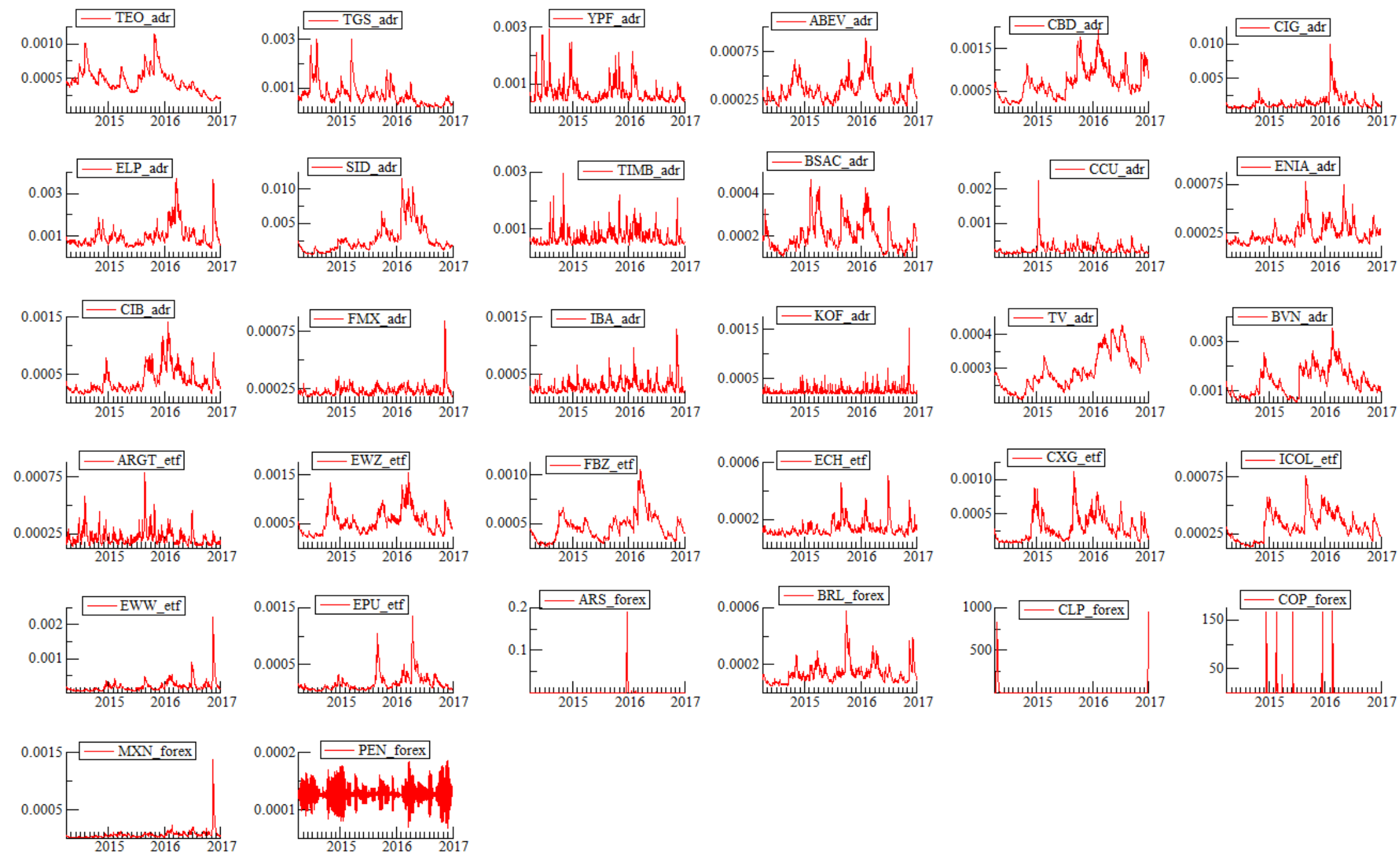

\section{Figure 2: Conditional variance (Brazilian crisis period)}




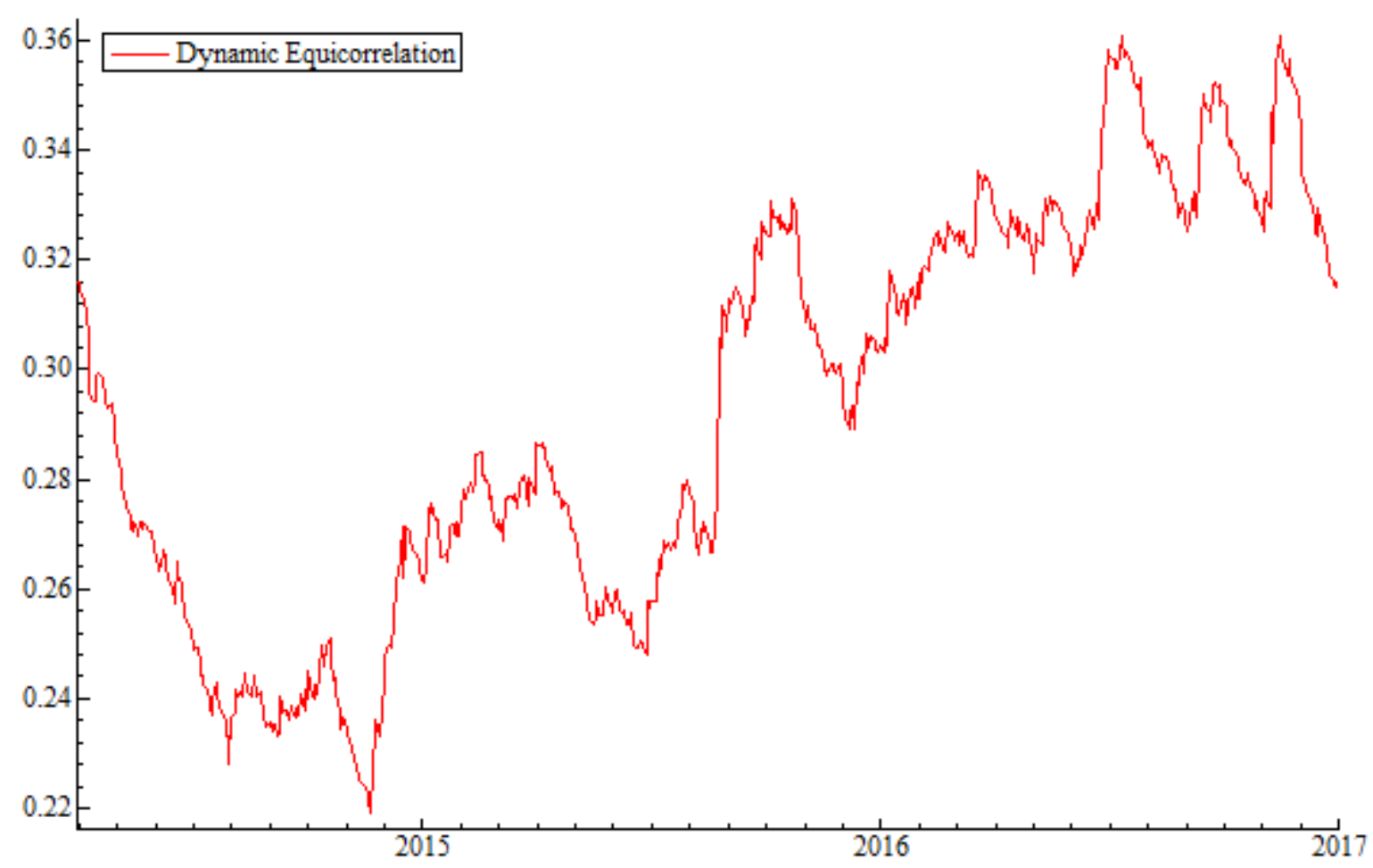

Figure 3: Conditional correlation (Brazilian crisis period)

Source: Authors' own computations

Table 4(c) presents the results of ARMA-GARCH-DECO estimations during the calm period. The positivity constraint for the GARCH $(1,1)$ and the condition for existence of the fourth moment of the GARCH are both observed for assets related to defensive super sector (ABEV, CBD, ELP, SID, CCU, ENIA, FMX, IBA, KOF, TV), communication and financial services (TIMB, BSAC), exchange-traded funds (EWZ, FBZ, ECH, CXG, ICOL, EWW, EPU) and foreign exchange rate (BRL/USD, CLP/USD, MXN/USD, PEN/USD). Results also show that financial instruments emanating from energy and telecommunication-related sectors (TEO, TGS, YPF, CIG), Exchange traded fund (ARGT) and Forex (ARS/USD) observed the positivity constraint for the GARCH $(1,1)$ is but denied the condition for existence of the fourth moment. Moreover, their positive coefficient $\alpha$ from the fitted model capturing the influence of new shocks on volatility is statistically significant. Moreover, we find the condition for existence of the fourth moment of the GARCH is observed in CIB but the positivity constraint for the GARCH $(1,1)$ is not. That constraint is over 1. Additionally, Figure 4 plotting the conditional variance indicate a relatively stable price as low volatility is observed during that period. Figure 5 draws the conditional correlation during that crisis period. It can be seen that the constraints described above are satisfied for all possible realizations of the past information and for all linear combinations of the variables. Results for Dynamic equicorrelation model are also reported. Rho with a statistically significant score of 0.2450 shows that the assets ADRs, ETFs and Forex are less correlated with the market movements during calm period than during period of financial turmoil. Moreover, we also find that long-run volatility impact of Beta(DECO) (0.8962) is lower than that of other sub-periods, meaning that volatility is less persistent compared to other periods. 
Table 4(c): Summary Statistics of multivariate ARMA-GARCH-DECO Model (calm period)

\begin{tabular}{|c|c|c|c|c|c|c|c|}
\hline \multicolumn{8}{|c|}{ Univariate ARMA(1,1)-GARCH(1,1) model } \\
\hline Variable & Phi & \multicolumn{2}{|c|}{ Theta } & \multicolumn{2}{|c|}{ Alpha } & Beta & Log-likelihood \\
\hline TEO & -0.8685 *** & \multicolumn{2}{|c|}{$0.9000^{* * * *}$} & \multicolumn{2}{|c|}{0.4610} & 0.4459 & 1721.751 \\
\hline TGS & -0.5406 & 0.6465 & & \multicolumn{2}{|c|}{$0.9808 *$} & 0.1405 & 1517.608 \\
\hline YPF & $-0.5735 * * *$ & \multicolumn{2}{|c|}{$0.6178 * *$} & \multicolumn{2}{|c|}{$0.5018 *$} & 0.2124 & 1716.452 \\
\hline ARGT & \begin{tabular}{l|l}
0.3851 \\
\end{tabular} & -0.4492 & & \multicolumn{2}{|l|}{0.3391} & 0.6303 & 2034.975 \\
\hline ARS-USD & -0.2187 & \multicolumn{2}{|c|}{$0.6375 * * *$} & \multicolumn{2}{|c|}{$0.9609 \mid * *$} & $0.4445^{* * *}$ & 2370.064 \\
\hline $\mathrm{ABEV}$ & -0.0219 & -0.0412 & & \multicolumn{2}{|c|}{0.0089} & $0.9822^{* * * *}$ & 1959.488 \\
\hline CBD & -0.1664 & 0.2053 & & \multicolumn{2}{|l|}{0.1303} & \begin{tabular}{l|l}
0.2017 \\
\end{tabular} & 1770.387 \\
\hline CIG & -0.0048 & -0.0654 & & \multicolumn{2}{|c|}{0.0701} & $0.9256 * * *$ & 1598.772 \\
\hline ELP & 0.2904 & -0.3136 & & \multicolumn{2}{|c|}{$0.0816 *$} & $0.8407^{* * *}$ & 1745.993 \\
\hline SID & -0.1195 & 0.0474 & & \multicolumn{2}{|c|}{0.0094} & $0.9834 * * *$ & 1465.927 \\
\hline TIMB & $0.4428 *$ & $-0.4995 *$ & & \multicolumn{2}{|l|}{0.0882} & $0.6804^{*}$ & 1896.450 \\
\hline EWZ & 0.1864 & -0.2417 & & \multicolumn{2}{|l|}{0.2499} & 0.3577 & 1939.807 \\
\hline FBZ & $0.5546 * *$ & $-0.5725^{*}$ & & \multicolumn{2}{|c|}{0.2950} & \begin{tabular}{|l|l|}
0.0261 & \\
\end{tabular} & 1932.067 \\
\hline BRL/USD & -0.2150 & 0.0272 & & \multicolumn{2}{|c|}{$0.1836 *$} & $0.6587^{* * *}$ & 2452.110 \\
\hline BSAC & 0.4078 & -0.4328 & & \multicolumn{2}{|c|}{$0.3585 * *$} & \begin{tabular}{l|l}
0.2137 \\
\end{tabular} & 2171.146 \\
\hline CCU & -0.0967 & 0.1169 & & \multicolumn{2}{|c|}{0.0716 * } & $0.7627^{* * *}$ & 2251.917 \\
\hline ENIA & -0.1162 & 0.1028 & & \multicolumn{2}{|c|}{$0.0673 *$} & $0.5793^{* * *}$ & 2063.351 \\
\hline $\mathrm{ECH}$ & $\begin{array}{l}-0.6769 \\
\end{array}$ & $0.7632 *$ & & \multicolumn{2}{|c|}{\begin{tabular}{l|l|l}
$0.1498 * *$ \\
\end{tabular}} & $0.7939 * * *$ & 2272.495 \\
\hline CLP/USD & \begin{tabular}{|c|c|}
-0.1122 \\
\end{tabular} & -0.0652 & & \multicolumn{2}{|c|}{$0.1962 * * *$} & $0.6503^{* * * *}$ & 2550.393 \\
\hline CIB & $0.5994 * * *$ & $-0.5750 *$ & $* * *$ & 0.1283 & $* *$ & -0.3216 & 2056.856 \\
\hline $\mathrm{CXG}$ & 0.6675 *** & $-0.5859 *$ & $* * *$ & 0.0817 & $* *$ & $0.8349 * * *$ & 2343.226 \\
\hline ICOL & $0.6821^{* * *}$ & $-0.6145 *$ & $* * *$ & 0.0707 & $* *$ & $0.8907^{* * * *}$ & 2359.578 \\
\hline COP/USD & $\mathrm{nc}$ & $\mathrm{nc}$ & & $\mathrm{nc}$ & & $\mathrm{nc}$ & $\mathrm{nc}$ \\
\hline FMX & $0.56011^{* * *}$ & $-0.6195 *$ & $* * *$ & 0.0683 & $* *$ & 0.8816 *** & 2184.295 \\
\hline IBA & 0.0551 & -0.2197 & & 0.0399 & & $0.8445^{* * * *}$ & 2023.665 \\
\hline $\mathrm{KOF}$ & $-0.9790 * * *$ & $0.9897 *$ & $* * *$ & 0.0484 & $* *$ & $0.8910^{* * * *}$ & 2140.843 \\
\hline TV & \begin{tabular}{l|l}
0.2632 \\
\end{tabular} & -0.1877 & & 0.0245 & $*$ & $0.9634^{* * *}$ & 1917.717 \\
\hline EWW & 0.6953 *** & $-0.7109 *$ & $* * *$ & 0.1161 & $* * *$ & $0.8034 * * *$ & 2227.390 \\
\hline MXN/USD & \begin{tabular}{l|l}
0.4707 \\
\end{tabular} & -0.5074 & & 0.0993 & $* *$ & $0.8450^{* * *}$ & 2703.962 \\
\hline $\mathrm{BVN}$ & $\mathrm{nc}$ & $\mathrm{nc}$ & & $\mathrm{nc}$ & & $\mathrm{nc}$ & $\mathrm{nc}$ \\
\hline EPU & $0.7841^{* * *}$ & $-0.7234 *$ & $* * *$ & 0.0823 & $* * *$ & $0.7480^{* * * *}$ & 2485.406 \\
\hline PEN/USD & |0.0861 & $-0.6636 *$ & $* * *$ & 0.0567 & & 0.1323 & 2342.610 \\
\hline & & ynamic E & Equi & prrelatio & on $M$ & & \\
\hline Rho & 0.2450 & & & & & & \\
\hline $\begin{array}{l}\text { Alpha } \\
\text { (DECO) }\end{array}$ & 0.0284 & & & & & & \\
\hline Beta (DECO) & 0.8962 & & & & & & \\
\hline Df & 10.5937 & & & & & & \\
\hline AIC & -185.4022 & & & & & & \\
\hline $\begin{array}{l}\text { Log- } \\
\text { likelihood }\end{array}$ & 70092.645 & & & & & & \\
\hline
\end{tabular}

Note: $* * *$ and $* * *$ are significance at 10,5 and $1 \%$ levels; nc means that the tests are not reported since there is no convergence (no improvement in line search) using numerical derivatives. Df refers to degrees of freedom and AIC means Akaike information criterion.

Source: Authors' own computations 

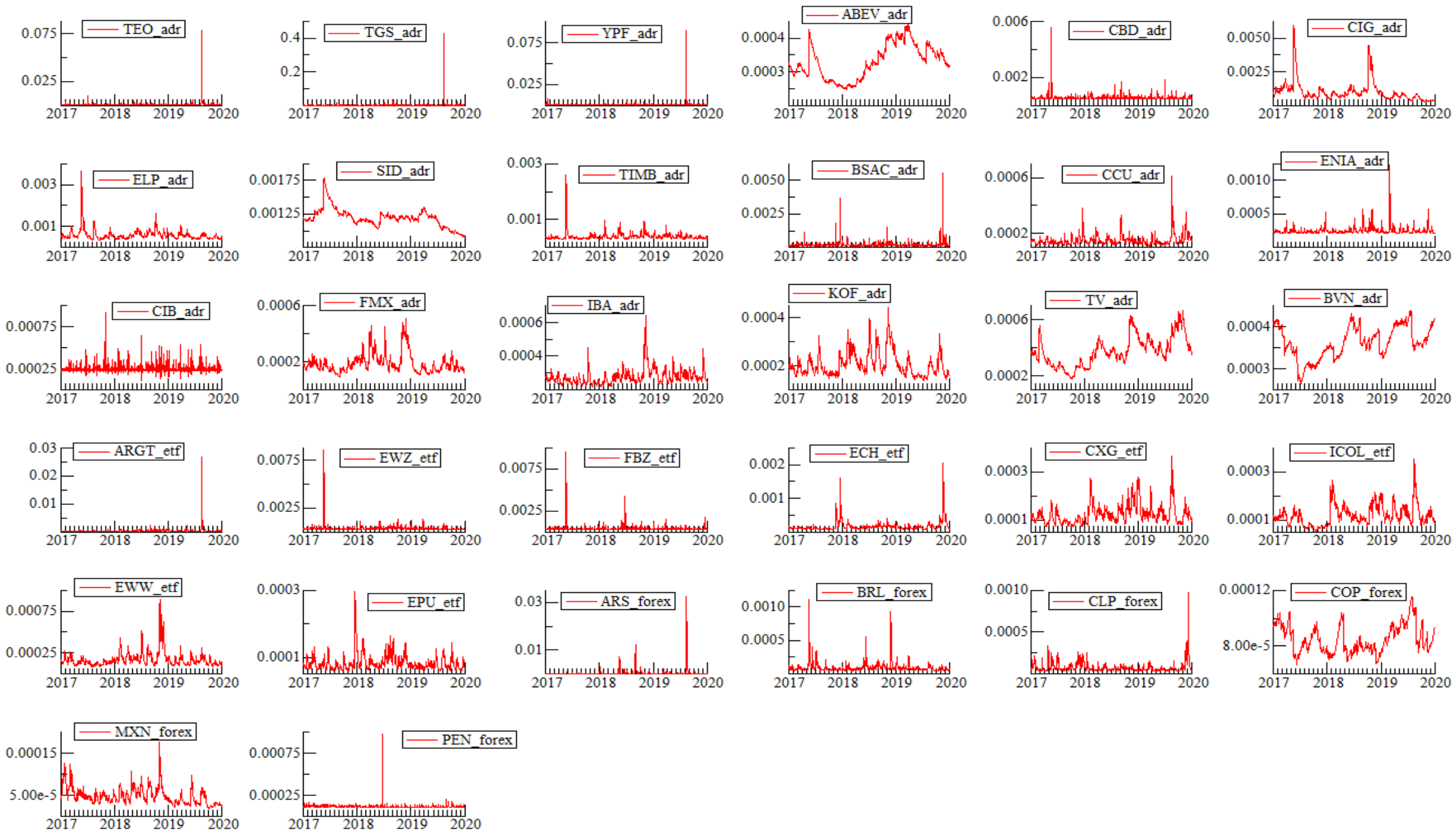

Figure 4: Conditional variance (calm period)

Source: Authors' own computations 


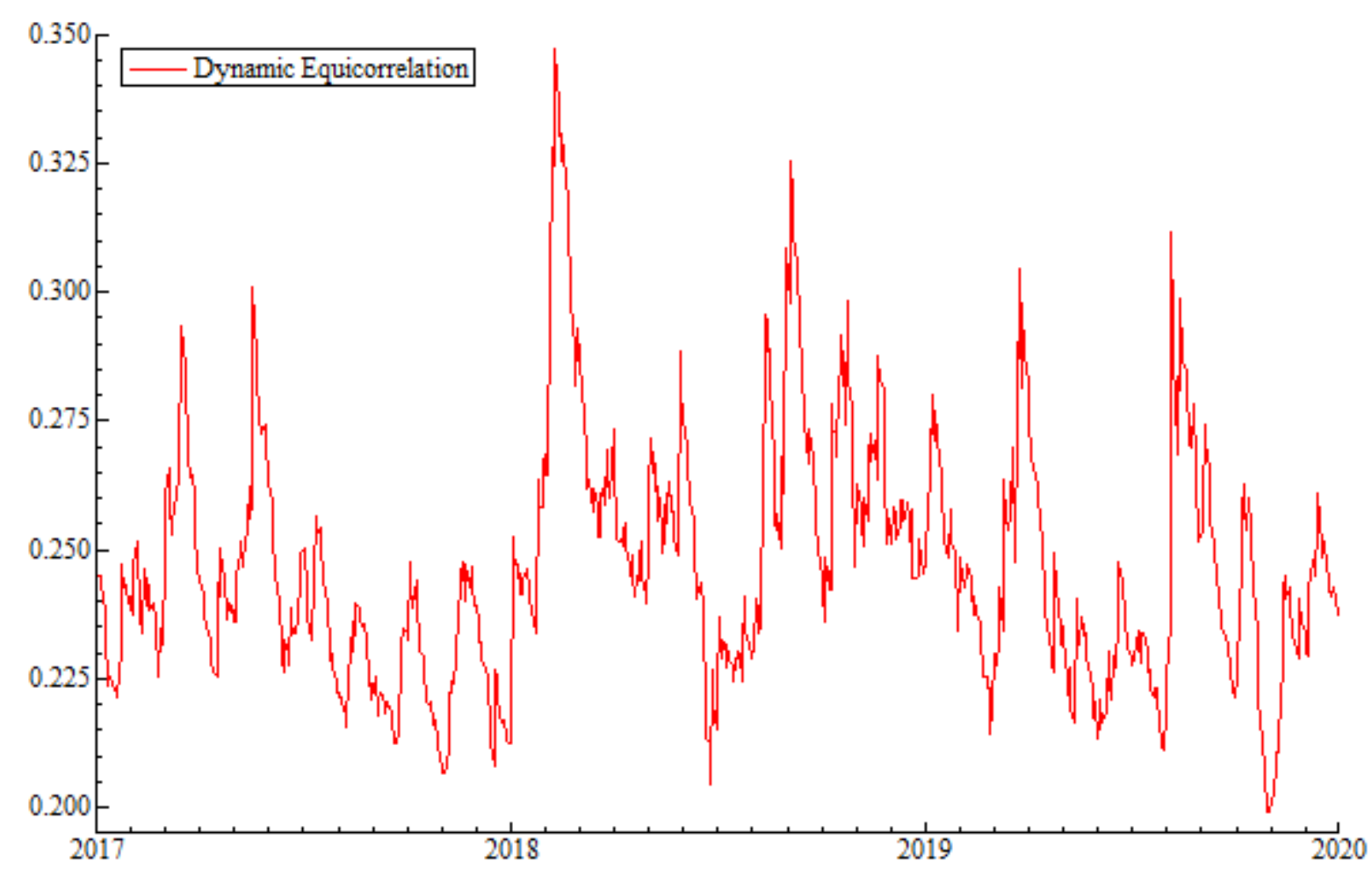

Figure 5: Conditional correlation (calm period)

Source: Authors' own computations

We ran ARMA-GARCH-DECO estimations for the period, we named "COVID-19 recession period". Table 4(d) presents the results of those estimations. Volatility persistence is observed in our data during that period as shown by the existence of the positivity constraint and the fourth moment of the GARCH in assets related to defensive super sector (ABEV, CCU and IBA, ENIA, CBD), communication and financial services (TEO, TV, BSAC,), exchangetraded funds (ARGT, ECH) and Foreign exchange rate (ARS/USD, BRL/USD COP/USD). This implies that covid-19 shocks have long standing effects on many industries such as department stores, telecom services, banks, food and beverage. However, we only observed the positivity constraint for the GARCH $(1,1)$ in assets returns of different sectors such as energy and telecommunication services (TGS, YPF, TIMB, CIB), utilities and basic materials (CIG, ELP, SID, FMX, KOF, BVN), ET funds (EWZ, CXG, ICOL, EWW, EPU) and forex (MXN/USD, PEN/USD). The condition for existence of the fourth moment of the GARCH is not observed in the above-mentioned financial returns. High value of $\beta$ indicates that old shocks tend to persist. Figure 5 indicates a relatively high volatility during the stock market crash period in all markets while Figure 6 translates how highly correlated those markets are during that period. Dynamic Equicorrelation Model with significant degrees of freedom (10.5937) is also reported. Coefficient rho (0.3032) shows a relatively high level of correlation among financial assets compared to the calm period. Beta (DECO) (0.8906) measuring the long-run volatility effect confirming the long impacts of the novel coronavirus pandemic on those financial instruments. 
Table 4(d): Summary Statistics of multivariate ARMA-GARCH-DECO Model (COVID-19 recession period)

\begin{tabular}{|c|c|c|c|c|c|c|c|c|c|}
\hline \multicolumn{10}{|c|}{ Univariate ARMA(1,1)-GARCH(1,1) model } \\
\hline Variable & \multicolumn{2}{|c|}{ Phi } & \multicolumn{2}{|c|}{ Theta } & \multicolumn{2}{|c|}{ Alpha } & \multicolumn{2}{|l|}{ Beta } & \multirow{2}{*}{$\begin{array}{c}\text { Log-likelihood } \\
530.631\end{array}$} \\
\hline TEO & 0.1985 & & $-0.2363 *$ & & \multicolumn{2}{|c|}{0.1574} & 0.7273 & **** & \\
\hline TGS & 0.2708 & & -0.3825 & & \multicolumn{2}{|l|}{0.2552} & 0.7143 & $* *$ & 523.509 \\
\hline YPF & -0.3791 & & 0.3105 & & \multicolumn{2}{|c|}{$0.4247^{*}$} & 0.5637 & $* * *$ & 457.357 \\
\hline ARGT & $-0.6425 *$ & *** & $0.5426^{*}$ & $* * *$ & \multicolumn{2}{|c|}{$0.1874 *$} & 0.7564 & $* * *$ & 642.046 \\
\hline ARS/USD & 0.1577 & & $-0.4166^{*}$ & $* * *$ & \multicolumn{2}{|c|}{0.1201} & 0.5983 & $* *$ & 1322.303 \\
\hline ABEV & $-0.5483 *$ & $* * *$ & $0.4314^{*}$ & & \multicolumn{2}{|c|}{0.1733 **** } & $0.7457=$ & $* * *$ & 533.089 \\
\hline CBD & $-0.5367 *$ & $* * *$ & $0.4134 *$ & $* * *$ & \multicolumn{2}{|c|}{0.2193} & 0.3288 & & -164.080 \\
\hline CIG & -0.3156 & & 0.2920 & & \multicolumn{2}{|l|}{0.2360} & $0.7226=$ & $* * *$ & 497.919 \\
\hline ELP & 0.3007 & & $-0.3779 *$ & & \multicolumn{2}{|c|}{$0.2417^{* * * *}$} & $0.7127=$ & $* * *$ & 549.641 \\
\hline SID & -0.3170 & & 0.2282 & & \multicolumn{2}{|c|}{\begin{tabular}{l|l}
0.3171 \\
\end{tabular}} & 0.5998 & & 443.088 \\
\hline TIMB & -0.0645 & & 0.0034 & & \multicolumn{2}{|c|}{$0.2375^{* *}$} & 0.7478 & $* * *$ & 555.588 \\
\hline EWZ & $-0.4654 *$ & & 0.3042 & & \multicolumn{2}{|c|}{$0.3640^{* * * *}$} & $0.5870=$ & $* * *$ & 584.899 \\
\hline FBZ & $-0.4851 *$ & & $0.3981 *$ & & \multicolumn{2}{|c|}{$0.3601^{* * * *}$} & $0.5484=$ & $* * *$ & 587.643 \\
\hline BRL/USD & -0.0061 & & -0.0894 & & \multicolumn{2}{|c|}{$0.1704 * *$} & $0.7905=$ & $* * *$ & 793.596 \\
\hline BSAC & $-0.7587^{*}$ & $* * *$ & $0.6842 *$ & $* * *$ & \multicolumn{2}{|c|}{$0.3960^{* *}$} & 0.4085 & $*$ & 590.566 \\
\hline CCU & -0.0739 & & 0.0604 & & 0.1883 & & 0.7418 & $* * *$ & 652.918 \\
\hline ENIA & 0.3405 & & -0.1996 & & 0.2315 * & $* * *$ & $0.7124=$ & $* * *$ & 633.365 \\
\hline $\mathrm{ECH}$ & -0.2398 & & 0.2241 & & \multicolumn{2}{|c|}{$0.2394 * * *$} & $0.6389=$ & $* * *$ & 655.043 \\
\hline CLP/USD & $\mathrm{nc}$ & & $\mathrm{nc}$ & & $\mathrm{nc}$ & & $\mathrm{nc}$ & & $\mathrm{nc}$ \\
\hline CIB & 0.4328 & & -0.3528 & & 0.4585 & & $0.5532=$ & $* * *$ & 557.354 \\
\hline$\overline{\mathrm{CXG}}$ & $0.5350 *$ & & -0.3310 & & 0.4677 & & $0.5446=$ & $* * *$ & 670.106 \\
\hline ICOL & $0.6165^{*}$ & & $-0.4835^{*}$ & $* * *$ & $0.6144=$ & & $0.4390=$ & $* *$ & 675.645 \\
\hline COP/USD & $0.7147 *$ & & $-0.6478 *$ & $* * *$ & 0.1443 & & $0.8282=$ & $* *$ & 872.060 \\
\hline FMX & $-0.4706^{*}$ & & $0.3854^{*}$ & & 0.3053 & $* *$ & $0.6491=$ & $* * *$ & 637.393 \\
\hline IBA & $-0.6002 *$ & & $0.5171 *$ & $* * *$ & $0.2455=$ & $* *$ & $0.6756=$ & $* * *$ & 653.380 \\
\hline KOF & $-0.5965 *$ & & $0.4787 *$ & $* * *$ & 0.3141 & $* * *$ & $0.6488=$ & $* * *$ & 670.205 \\
\hline TV & $-0.5742 *$ & & $0.5063 *$ & $* * *$ & 0.1708 & $* *$ & $0.7577=$ & $* * *$ & 497.906 \\
\hline EWW & $-0.5846 *$ & & $0.4858 *$ & $* * *$ & 0.2958 & $* * *$ & $0.6779=$ & $* * *$ & 658.955 \\
\hline MXN/USD & $-0.4559 *$ & & $0.4028 *$ & & 0.2499 & $* *$ & $0.7432=$ & $* * *$ & 855.085 \\
\hline $\mathrm{BVN}$ & $0.4907 *$ & & $-0.5809 *$ & $* * *$ & $0.3050=$ & $* * *$ & 0.6338 & $* * *$ & 534.499 \\
\hline EPU & $-0.5509 *$ & & $0.4802 *$ & $* * *$ & 0.3159 & $* * *$ & $0.6296=$ & $* * *$ & 732.553 \\
\hline PEN/USD & $-0.6311 *$ & & 0.1738 & & 0.3064 & & 0.7392 & $* * *$ & 866.887 \\
\hline & & & Dynamic & Equ & orrelatio & on $\mathrm{M}$ & & & \\
\hline Rho & & & $.3032^{* * *}$ & & & & & & \\
\hline Alpha (DEC & & & .0123 & & & & & & \\
\hline Beta (DECO & & & $.8906 * * *$ & & & & & & \\
\hline Df & & & $8101^{* * * *}$ & & & & & & \\
\hline AIC & & & \begin{tabular}{l|l}
.0796 & \\
\end{tabular} & & & & & & \\
\hline Log-likeliho & & 225 & 0.819 & & & & & & \\
\hline
\end{tabular}

Note: $* * *$ and $* * *$ are significance at 10,5 and $1 \%$ levels; nc means that the tests are not reported since there is no convergence (no improvement in line search) using numerical derivatives. Df refers to degrees of freedom and AIC means Akaike information criterion.

Source: Authors' own computations 

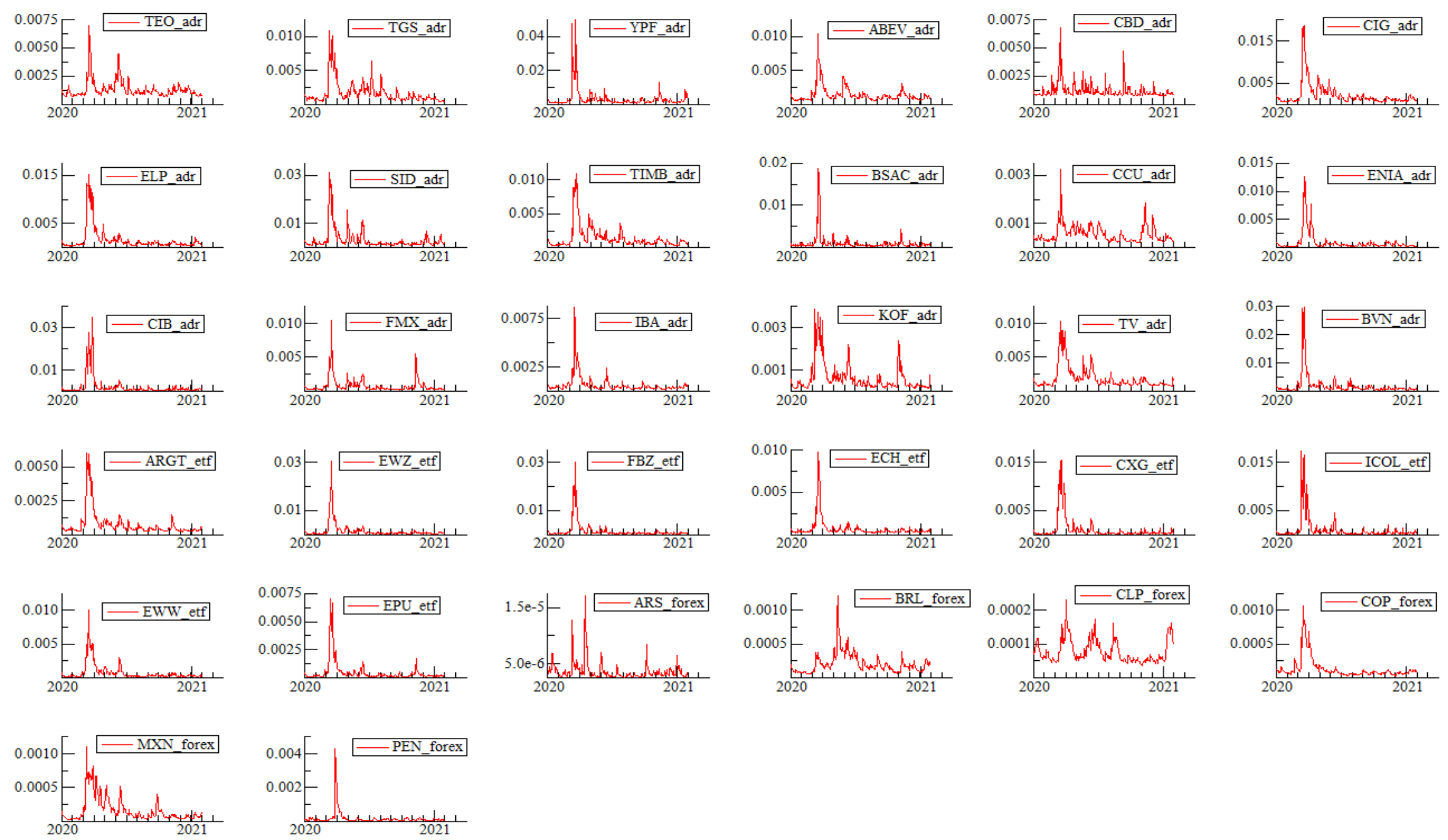

Figure 6: Conditional variance (COVID-19 recession period)

Source: Authors' own computations 


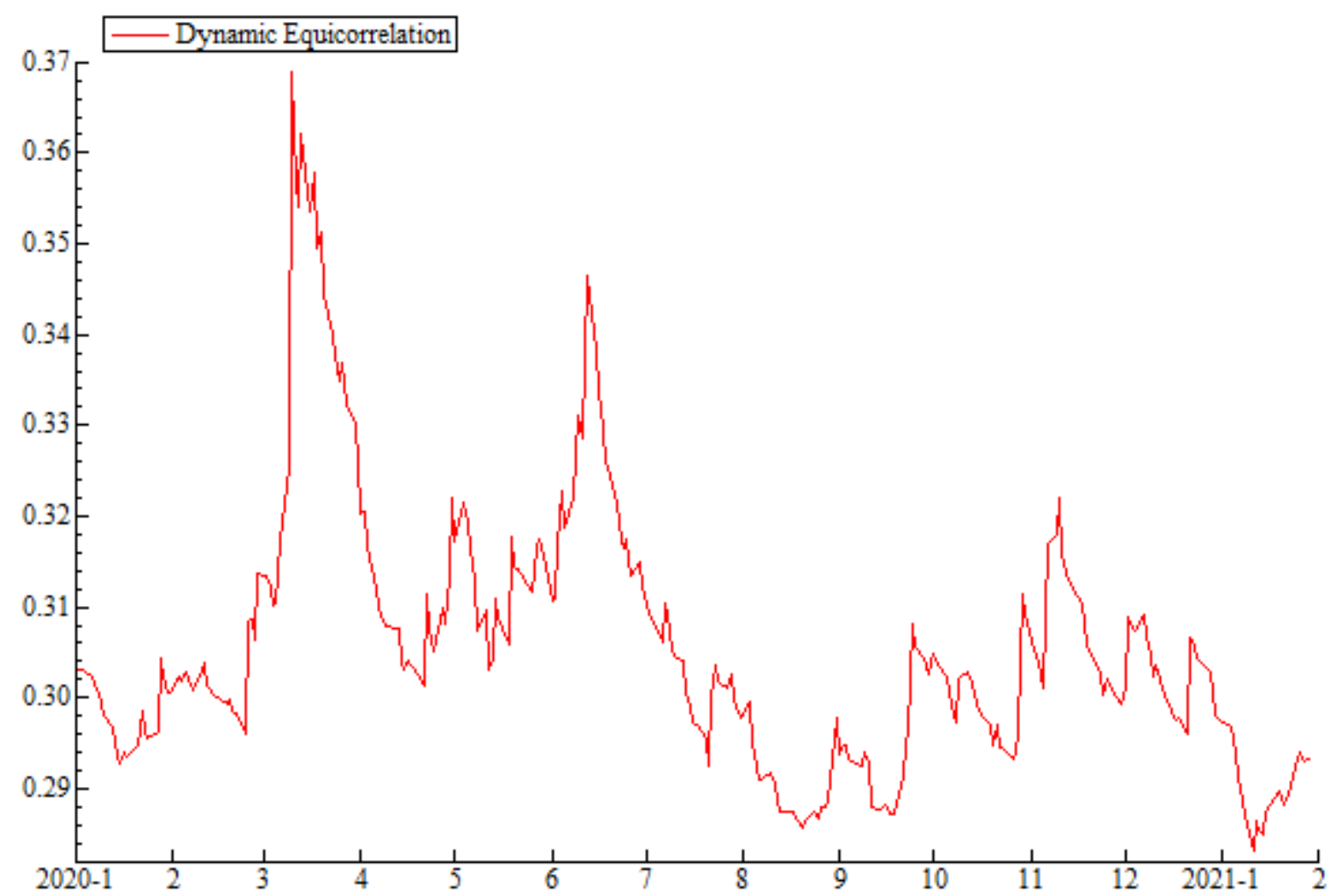

Figure 7: Conditional correlation (COVID-19 recession period)

Source: Authors' own computations

\section{Conclusion}

This paper uses ARMA-GARCH-DECO model to capture the impact of financial turmoil on 32 Latin American financial instruments including American depository receipt (ADR), Exchange-traded fund (ETF) and forex. For that purpose, we divided our sample into full sample, Brazilian crisis, calm period and COVID-19 recession period. In the overall sample, our model finds short-term properties in the volatility of most instruments including those belonging to defensive super sector. Moreover, statistically significant coefficients of the dynamic equicorrelation model show the presence of long-run volatility impact among the markets implying the predictability in the dispersion structure of returns. Defensive super sector and basic materials are the most impacted sectors during economic crises. The evidence presented here indicates that in times of economic disruption like in the midst of the COVID19 pandemic, Latin American financial instruments do not act as safe harbor investments.

Other interesting finding is related to the correlation among markets. Results also show that financial assets are relatively more correlated during period of financial crises than in normal periods. This effect is particularly persistent during COVID-19 pandemic, lessening the benefits of international portfolio diversification for investors. Those results are of potential interest to various economic agents including international investors and policymakers who look a better understanding of the dynamics of spillovers among the behavior of emerging financial assets in order to build efficient risk hedging models or to implement appropriate policies to react to information transmission in periods of financial turmoil. This gives them the opportunity to build new diversification strategies in times of turbulence and design a better decision model that can protect them against the risk of contagion. 
Although we have shown important contribution to the literature, it's crucial to notice some limitations that future studies can consider. First, the paper only used data related to ADRs, ETF and Forex in 6 Latin American countries, meaning that our sample may not be representative of all markets instruments data related to that particular region. Future work can extend this framework by considering data from other institutions and markets. Second, we restricted our models in considering data and information during two recent crises. Subsequent research can enlarge the total observations by considering a longer period of time. Lastly, it appears that ARMA $(1,1)$-GARCH $(1,1)$ models are too simple and may be inappropriate in correcting simultaneously serial correlation problems among series. other econometric methods other FI models like HYGARCH and FIEGARCH can be applied to these financial derivative instruments to determine other aspects of the long-memory and leverage effects phenomena. These limitations can provide future research avenues and can step on the contributions established by this paper regarding the effect of economic crisis on emerging markets instruments.

\section{References}

[1] Ali, M., Alam, N., and Rizvi, S. A. R. (2020). Coronavirus (COVID-19)—An epidemic or pandemic for financial markets. Journal of Behavioral and Experimental Finance, 27, 100341.

[2] Ali, M., Alam, N., and Rizvi, S. A. R. (2020). Coronavirus (COVID-19)—An epidemic or pandemic for financial markets. Journal of Behavioral and Experimental Finance, 27, 100341.

[3] Bollerslev, T. (1987). A conditionally heteroskedastic time series model for speculative prices and rates of return. The review of economics and statistics, 542-547.

[4] Cai, X. J., Tian, S., and Hamori, S. (2016). Dynamic correlation and equicorrelation analysis of global financial turmoil: Evidence from emerging East Asian stock markets. Applied Economics, 48(40), 3789-3803.

[5] Choudhry, T., Hassan, S. S., and Shabi, S. (2015). Relationship between gold and stock markets during the global financial crisis: Evidence from nonlinear causality tests. International Review of Financial Analysis, 41, 247-256.

[6] Costa Correa, A. C., Pimenta Júnior, T., and Eduardo Gaio, L. (2018). Interdependence and asymmetries: Latin American ADRs and developed markets. Brazilian Business Review (English Edition), 15(4).

[7] Danielsson, J. (2011). Financial risk forecasting: the theory and practice of forecasting market risk with implementation in R and Matlab (Vol. 588): John Wiley \& Sons.

[8] Diamandis, P. F. (2009). International stock market linkages: Evidence from Latin America. Global Finance Journal, 20(1), 13-30.

[9] Doornik, J. A., Hendry, D. F., and Shephard, N. (2002). Computationally intensive econometrics using a distributed matrix-programming language. Philosophical Transactions of the Royal Society of London. Series A: Mathematical, Physical and Engineering Sciences, 360(1795), 1245-1266.

[10] Engle, R., and Kelly, B. (2012). Dynamic equicorrelation. Journal of Business \& Economic Statistics, 30(2), 212-228.

[11] Engle, R. F. (1982). Autoregressive conditional heteroscedasticity with estimates of the variance of United Kingdom inflation. Econometrica: Journal of the econometric society, 987-1007.

[12] Escanciano, J. C., and Hualde, J. (2019). Measuring asset market linkages: Nonlinear dependence and tail risk. Journal of Business \& Economic Statistics, 1-25. 
[13] Esqueda, O. A., and Jackson, D. O. (2012). Currency depreciation effects on ADR returns: evidence from Latin America. Journal of Economics and Finance, 36(3), 691-711.

[14] Esqueda, O. A., Luo, Y., and Jackson, D. O. (2015). The linkage between the US "fear index" and ADR premiums under non-frictionless stock markets. Journal of Economics and Finance, 39(3), 541-556.

[15] Fama, E. F. (1965). The behavior of stock-market prices. The journal of Business, 38(1), 34-105.

[16] Ferraresi, M., Kotsogiannis, C., Rizzo, L., and Secomandi, R. (2020). The 'Great Lockdown'and its determinants. Economics letters, 197, 109628.

[17] Gamba-Santamaria, S., Gomez-Gonzalez, J. E., Hurtado-Guarin, J. L., and Melo-Velandia, L. F. (2017). Stock market volatility spillovers: Evidence for Latin America. Finance Research Letters, 20, 207-216.

[18] Hwang, J.-K. (2014). Spillover effects of the 2008 financial crisis in Latin America stock markets. International Advances in Economic Research, 20(3), 311-324.

[19] Kang, S. H., McIver, R., and Yoon, S.-M. (2017). Dynamic spillover effects among crude oil, precious metal, and agricultural commodity futures markets. Energy Economics, 62, 19-32.

[20] Ling, S., and McAleer, M. (2002). Stationarity and the existence of moments of a family of GARCH processes. Journal of Econometrics, 106(1), 109-117.

[21] Mandelbrot, B. B. (1997). The variation of certain speculative prices. In Fractals and scaling in finance (pp. 371-418): Springer.

[22] Marçal, E. F., Valls Pereira, P. L., Martin, D. M. L., and Nakamura, W. T. (2011). Evaluation of contagion or interdependence in the financial crises of Asia and Latin America, considering the macroeconomic fundamentals. Applied Economics, 43(19), 2365-2379.

[23] McKibbin, W., and Fernando, R. (2020). The global macroeconomic impacts of COVID19: Seven scenarios. Asian Economic Papers, 1-55.

[24] Nassif, A. (2017). An Analysis of Brazil's Economic Situation: 2014-2017, the short-term outlook and policy alternatives. Brazilian Keynesian Review, 3(1), 95-108.

[25] Rodriguez-Nieto, J. A., and Mollick, A. V. (2020). The US financial crisis, market volatility, credit risk and stock returns in the Americas. Financial Markets and Portfolio Management, 1-30.

[26] Topcu, M., and Gulal, O. S. (2020). The impact of COVID-19 on emerging stock markets. Finance Research Letters, 36, 101691.

[27] Tseng, J.-J., and Li, S.-P. (2011). Asset returns and volatility clustering in financial time series. Physica A: Statistical Mechanics and its Applications, 390(7), 1300-1314.

[28] Vartanian, P. R., and Garbe, H. d. S. (2019). The Brazilian economic crisis during the period 2014-2016: is there precedence of internal or external factors? Journal of International and Global Economic Studies, 12(1), 66-86.

[29] Yin, T., and Wang, Y. (2021). Market Efficiency and Nonlinear Analysis of Soybean Futures. Sustainability, 13(2), 518. 